\title{
Les paysages de champs surélevés de Guyane française : un patrimoine bioculturel menacé
}

Archaeological raised-field landscapes in French Guiana : a threatened biocultural heritage

\section{Stéphen Rostain et Doyle McKey}

\section{OpenEdition}

\section{Journals}

Édition électronique

URL : http://journals.openedition.org/ethnoecologie/2193

DOI : 10.4000/ethnoecologie.2193

ISSN : 2267-2419

Éditeur

Laboratoire Eco-anthropologie et Ethnobiologie

Référence électronique

Stéphen Rostain et Doyle McKey, « Les paysages de champs surélevés de Guyane française : un patrimoine bioculturel menacé », Revue d'ethnoécologie [En ligne], 7 | 2015, mis en ligne le 30 juin 2016, consulté le 20 avril 2019. URL : http://journals.openedition.org/ethnoecologie/2193 ; DOI : 10.4000/ ethnoecologie. 2193

Ce document a été généré automatiquement le 20 avril 2019

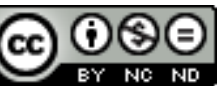

Revue d'ethnoécologie est mis à disposition selon les termes de la licence Creative Commons Attribution - Pas d'Utilisation Commerciale - Pas de Modification 4.0 International. 


\title{
Les paysages de champs surélevés de Guyane française : un patrimoine bioculturel menacé
}

\author{
Archaeological raised-field landscapes in French Guiana : a threatened
}

biocultural heritage

Stéphen Rostain et Doyle McKey

\section{Introduction}

\section{Le patrimoine bioculturel comme capital naturel}

1 Un des objectifs des efforts de conservation est de préserver le capital naturel représenté par la biodiversité et par les services fournis par les écosystèmes sains. Toutefois, le capital « naturel » n'est pas strictement biologique par nature, mais bien bioculturel. De nombreux paysages que l'on considère aujourd'hui comme méritant la préservation ont été façonnés en partie par des actions humaines passées (Rostain 1991, 2012a ; McKey et al . 2010). Ces mêmes actions ont laissé derrière elles des objets, des structures (parfois monumentales, parfois ordinaires), et même des éléments de paysages qui représentent un héritage culturel précieux, un lien avec les expériences du passé humain, enrichissant notre appréciation des paysages comme sources de beauté et marqueurs d'identité (Lipe 2002 ; Ryan 2009 ; Rostain 2014). 
Figure 1 : Champs surélevés précolombiens de Diamant, Guyane française

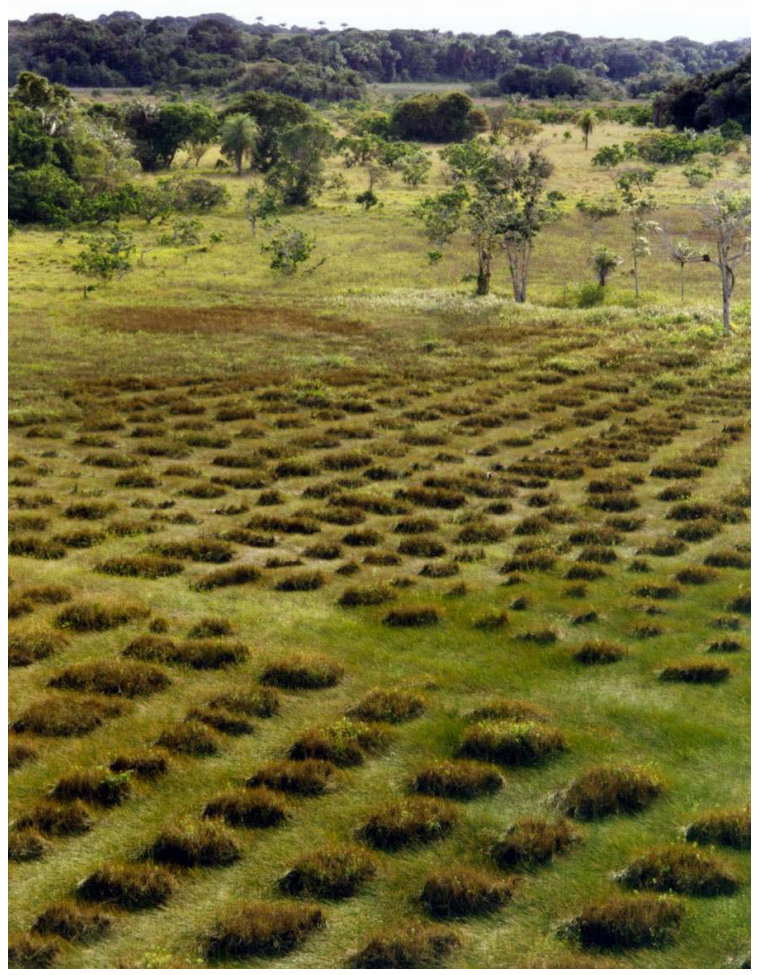

(c) S. Rostain

2 Les actions humaines passées ont également laissé derrière elles un autre type d'héritage: des altérations dans l'environnement qui ont des conséquences sur les processus écologiques, affectant parfois profondément le fonctionnement des écosystèmes (Briggs et al. 2006), et qui ont un impact pérenne et visible dans le paysage (Figure 1). Ces paysages altérés ont de la valeur en tant que capital naturel. Ils sont un guide pour interpréter les anciennes interactions homme/environnement. Les comprendre nous aide à intégrer l'écologie historique dans une base de connaissance nécessaire pour une conservation effective. La plupart des écosystèmes ont été substantiellement altérés par des activités humaines durant des décennies, des siècles ou plus encore. En fournissant un contexte écologique historique pour la conservation, la gestion et la restauration écologique, l'archéologie et l'histoire peuvent participer à l'établissement de points de comparaisons écologiques et de conditions de référence (Rick \& Lockwood 2013 ; Scharf 2014). L'étude de paysages altérés contribue également à nous enseigner comment les humains peuvent atteindre un certain degré de durabilité dans leur interaction avec les environnements (Fisher \& Feinman 2005; Hayashida 2005; Stump 2013).

3 La valeur conférée à certains paysages bioculturels les élève à un statut comparable à celui d'un monument. Par exemple, l'Organisation des Nations Unies pour l'Alimentation et l'Agriculture (FAO) reconnaît les «Systèmes Agricoles Traditionnels d'Importance Mondiale » (« Globally Important Agricultural Heritage Systems », GIAHS). Considérés comme le témoignage d'ingénieuses adaptations culturelles à des contraintes et des opportunités environnementales, les GIAHS sont définis comme «de remarquables systèmes d'utilisation de terres et de paysages riches dans leur diversité biologique globalement 
significative, émergeant de la co-adaptation d'une communauté avec son environnement et ses besoins et aspirations pour un développement durable » (Koohafkan \& Altieri 2011 : 1). Les GIAHS englobent des systèmes encore en usage aujourd'hui, qui sont néanmoins profondément ancrés dans une longue tradition. En Amérique Latine, cela comprend l'agriculture andine au Pérou, avec ses systèmes de terrasses pour contrôler l'appauvrissement des sols sur les pentes raides, et l'agriculture sur champs surélevés autour du lac Titicaca (Denevan 2001 ; Erickson 2003, 2006) ; l'agriculture en chinampas dans les lacs de la vallée centrale de Mexico (Niederberger Betton 1987); et l'agriculture sur terra preta ou Terres Noires Amazoniennes (Anthropogenic Dark Earths, ADE) (Teixeira et al. 2010) (Figures 2-4). Par exemple, ces dernières sont enrichies avec du charbon, résultant d'une longue occupation humaine. La forte teneur en charbon augmente considérablement leur capacité de rétention des éléments nutritifs, transformant les sols jaunes et rouges lourdement lessivés et acides, en terre hautement fertile (Glaser \& Birk 2012). Tous ces systèmes sont des exemples convaincants d'interaction durable de l'homme avec l'environnement, selon une trajectoire définie par l'adaptation et la résistance, plutôt que la dégradation. Ils sont envisagés comme des sources d'inspiration pour affronter le challenge de l'agriculture écologiquement durable au xxI ${ }^{e}$ siècle (Martin et al. 2010 ; Koohafkan \& Altieri 2011 ; Fuller \& Qingwen 2013).

Figure 2 : Les terrasses des Andes péruviennes, une technique agricole encore en pratique mais héritée d'une longue tradition précolombienne

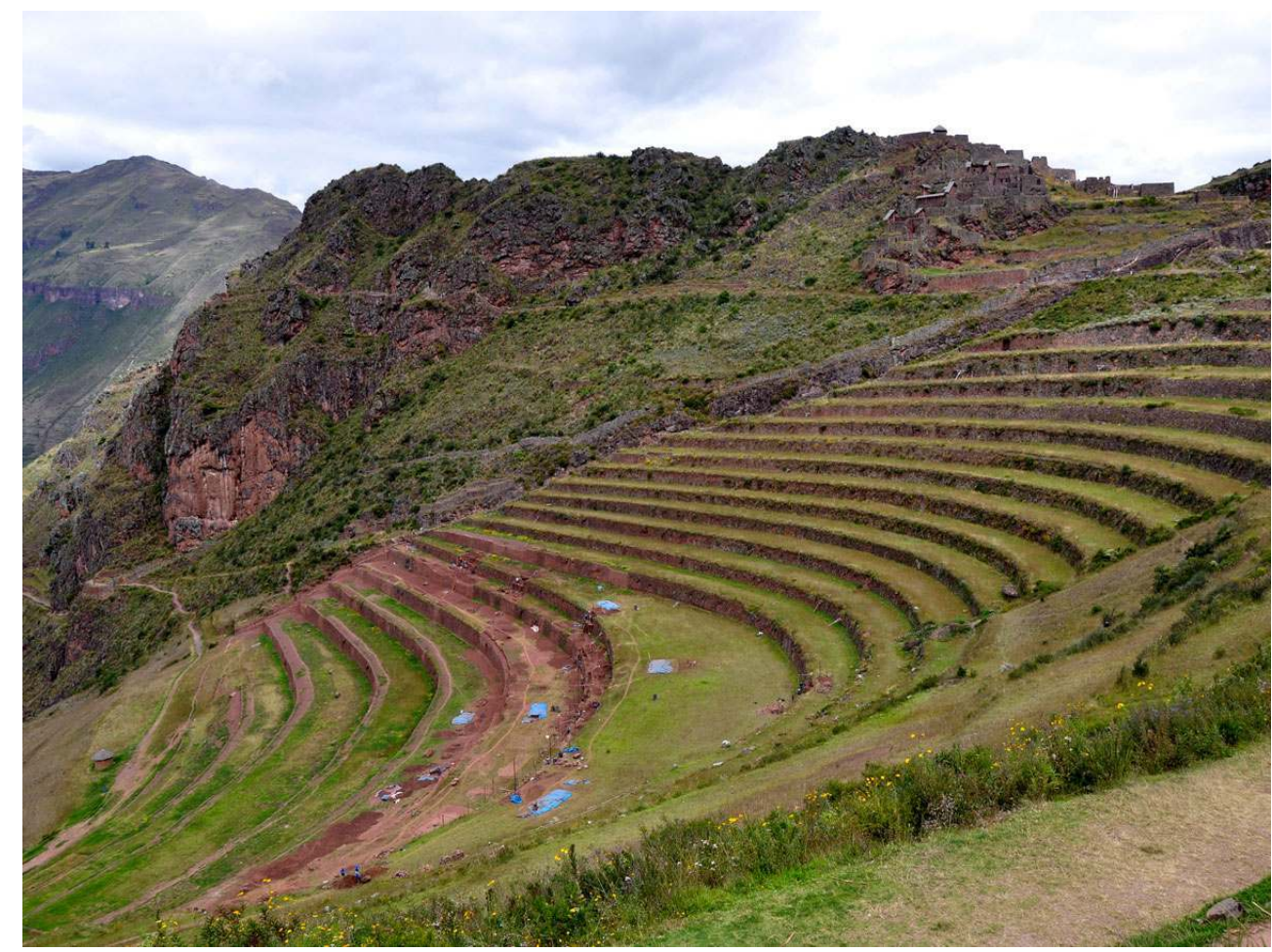

(c) S. Rostain 
Figure 3 : Les champs surélevés autour du lac Titicaca au Pérou, un autre système d'origine précolombienne

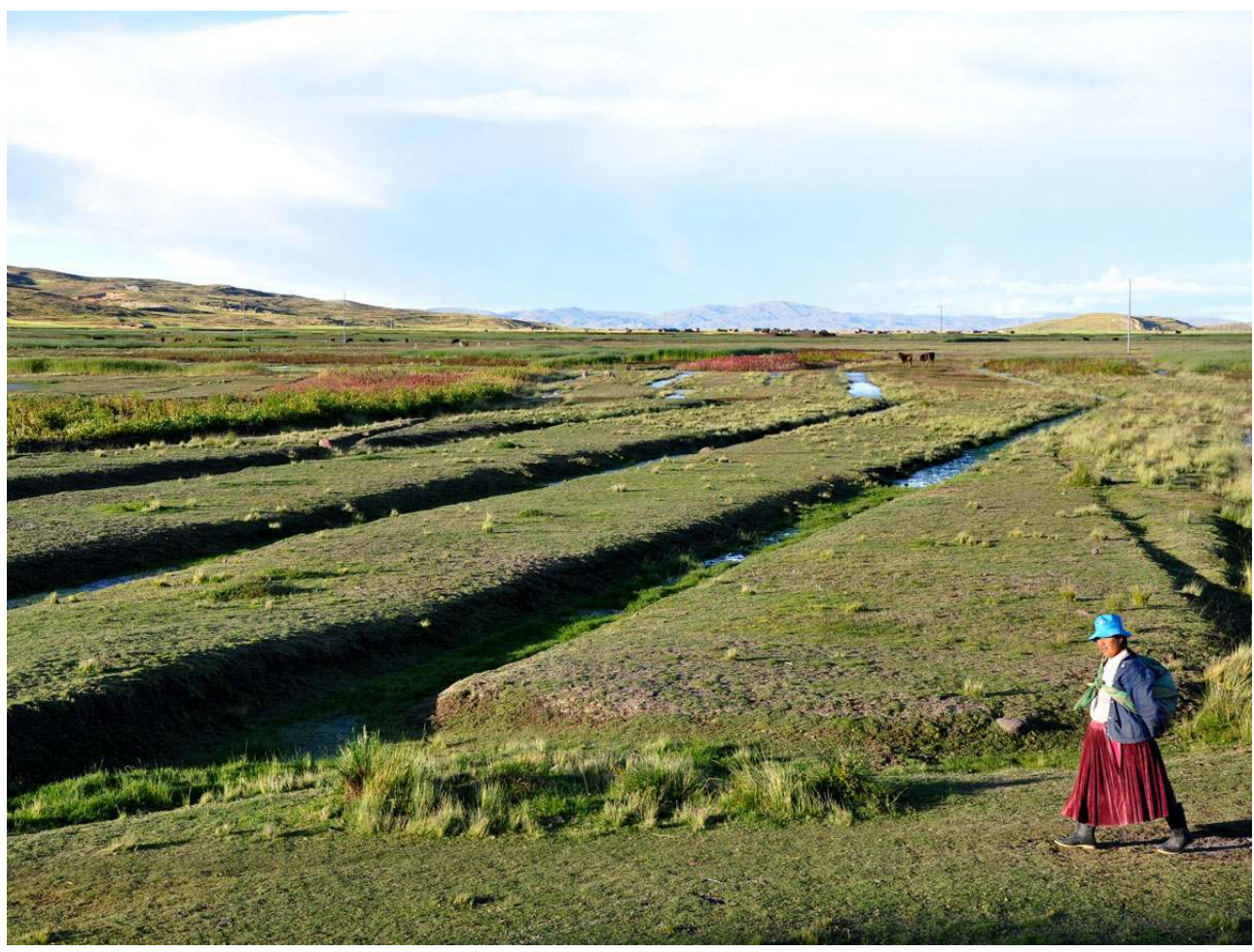

(c) S. Rostain

Figure 4 : Les chinampas dans le lac de Texcoco au Mexique, encore une technique agricole millénaire

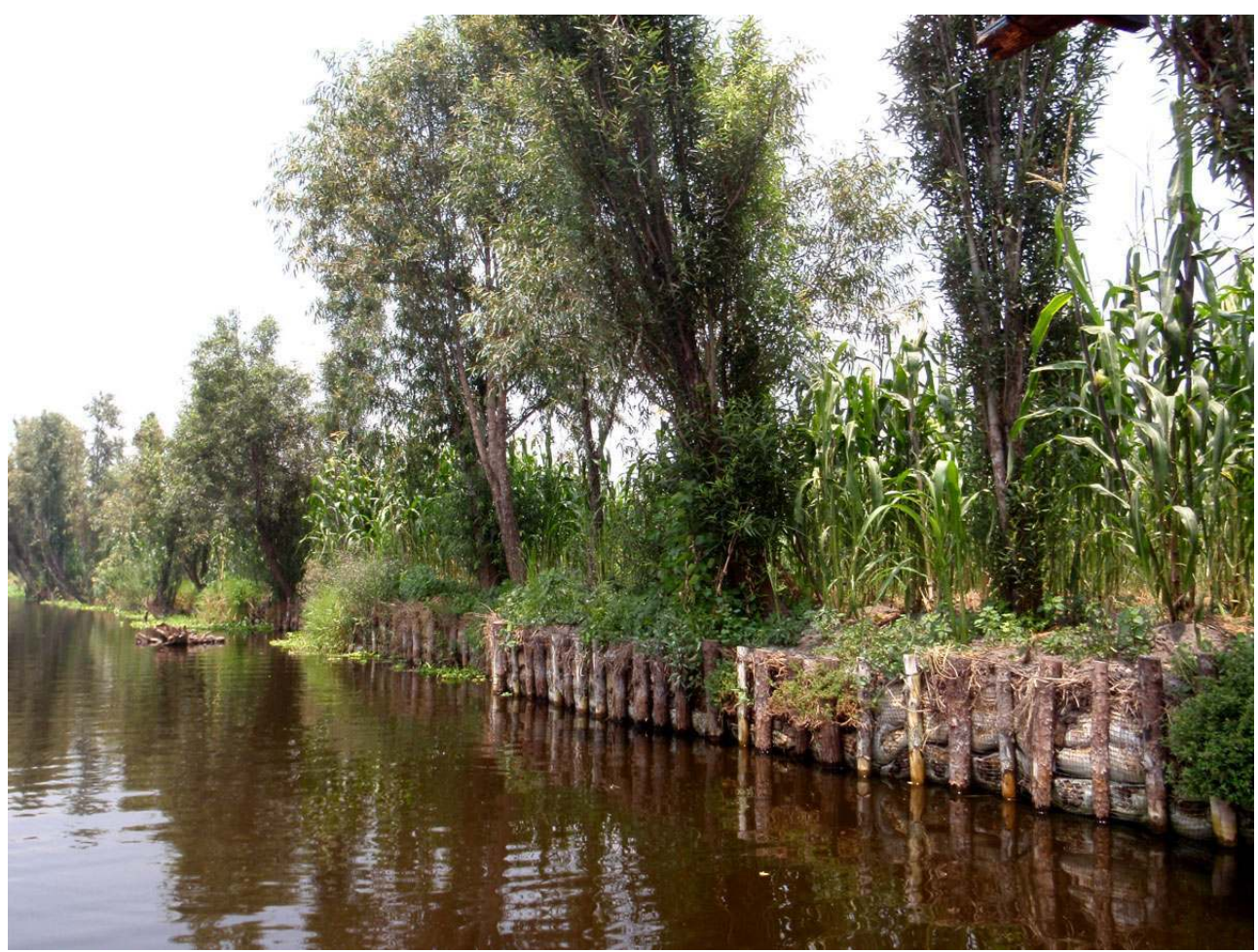

(c) S. Rostain 


\section{Les patrimoines bioculturels montrant des " histoires à succès » dans les relations homme/environnement sont-ils les seuls à mériter la préservation?}

Peu de personnes contesteraient l'intérêt de préserver les témoignages de telles « histoires à succès » dans les relations entre les humains et l'environnement. Dans de nombreux cas pourtant, la préoccupation de conserver les traces matérielles des altérations humaines passées sur le milieu est un sujet beaucoup plus problématique. Comme aujourd'hui, beaucoup de modifications humaines anciennes ont sans nul doute dégradé des environnements, et des paysages affectés par celles-ci sont des cibles plus appropriées à une cure ou une restauration écologique qu'à une préservation. Rares sont ceux à nier qu'il serait souhaitable de retirer les enrochements et le béton des berges autrefois couvertes de plantes et d'arbres afin de réhabiliter les cours d'eau (e.g. Fischenich 2003).

Mais alors que les Sites du Patrimoine Culturel de l'UNESCO magnifient non seulement la beauté (par exemple le Parc National de Tikal au Guatemala ou la ville de Quito en Équateur), mais aussi le mal absolu (comme le camp de concentration nazi d'AuschwitzBirkenau), la société pourrait bénéficier de la protection non seulement de témoignages du génie humain mais également des vestiges de la bêtise environnementale passée. L'étude de ces restes peut aider à comprendre les crises d'autrefois, ou même à réévaluer notre vision sur eux.

L'histoire bien connue du brusque effondrement écologique de l'île de Pâques, par exemple (Diamond 2005), s'est écroulée face au poids de nouvelles données archéologiques (Hunt 2006; Mulrooney 2013; Rull et al. 2013). La déforestation n'a pas mené à la famine et au cannibalisme, parce que l'agriculture continua à être productive dans le paysage de forêt largement grâce à la pratique polynésienne amplement répandue du défrichement lithique, utilisant les roches volcaniques de l'île (Baer et al. 2008), et selon certains auteurs (Hunt \& Lipo 2011) - parce qu'une espèce invasive introduite par l'homme, le rat polynésien, servait également comme source de protéines. Il doit être souligné en revanche que selon ces derniers auteurs, l'homme est toujours vu comme responsable de la dégradation de l'environnement de l'île, mais que cela prit plus de temps et que l'agent de déforestation a pu ne pas être l'abattage d'arbres par l'homme, mais la prédation de graines et de semis par les rats. Le point important ici est que les traces matérielles d'un impact humain hautement problématique sur l'environnement peuvent produire de riches leçons inattendues pour comprendre notre passé et gérer notre futur.

7 Les effets complexes de certaines altérations des paysages par l'Homme rendent l'estimation de leur juste valeur particulièrement ambiguë. Par exemple, les dépôts de résidus et de déchets miniers par l'activité des mines médiévales ont laissé des sols pollués avec des niveaux toxiques de métaux lourds. Mais, au cours des siècles, ces mines abandonnées ont également constitué des milieux où la sélection naturelle a favorisé l'émergence de plantes avec de nouvelles adaptations à la présence de ces métaux toxiques (Rascio \& Navari-Izzo 2011). Ces composants novateurs de la biodiversité (Barrutia et al. 2011) sont précieux pour la recherche fondamentale, en fournissant notablement des modèles biologiques pour l'étude de l'évolution en action. Ils ont également des applications pratiques, apportant de nouvelles ressources de valeur 
économique dans divers contextes, allant de l'utilisation dans la phytoremédiation (Vamerali et al. 2010) jusqu'au service comme nouvelles sources de catalyseurs industriels (Losfeld et al. 2014).

\section{Les vestiges de l'agriculture sur champs surélevés précolombiens comme patrimoine bioculturel}

\section{Les vestiges de champs surélevés comme capital naturel}

Des paysages caractérisés par des vestiges de champs surélevés portent le témoignage de l'histoire couplée des sociétés et des milieux le long de la côte des Guyanes (Rostain 1991, 2012a; McKey et al. 2010). Notre projet de recherche archéologique, mais surtout interdisciplinaire, mené la dernière décennie dans cette région a apporté un grand nombre de données et d'informations diverses sur l'impact humain ancien sur la bande littorale, documentant la transformation des paysages et l'occupation millénaire des lieux (Rostain 2008, 2012a; McKey et al. 2010). La présence de microfossiles distinctifs (phytolithes et grains d'amidon) montre l'importance ancienne du maïs (Iriarte et al. 2010). L'histoire environnementale des deux derniers millénaires, documentée par les phytolithes, pollens et charbons d'une carotte prise dans une tourbière près d'un site précolombien, suggère que les agriculteurs sur champs surélevés contrôlaient les incendies, pratiquant une gestion de type slash-and-mulch (couper-tailler) plutôt que slashand-burn (culture sur brûlis) (Iriarte et al. 2012).

Bien plus, les vestiges des champs surélevés ont une influence durable sur le fonctionnement des écosystèmes. Dès leur abandon par les agriculteurs, les champs surélevés furent remaniés par des organismes «ingénieurs du sol» tels les fourmis, termites, vers de terre et plantes (McKey et al. 2010 ; Renard et al. 2013). Ces organismes sont un sous-ensemble des organismes considérés par les écologues comme «ingénieurs d'écosystèmes ", c'est-à-dire, des organismes qui créent, maintiennent, modifient ou détruisent des habitats, affectant les conditions de vie pour eux-mêmes et pour d'autres organismes (Jones et al. 1994). Dans les sites que nous avons étudiés en Guyane, les activités des ingénieurs du sol sont concentrées dans les sols bien aérés des champs surélevés abandonnés. Dans ces anciens paysages agricoles, les nids des insectes sociaux se trouvent uniquement sur ces buttes car elles surplombent encore le niveau d'inondation. Les fourmis et termites sont des "central-place foragers ", qui ramènent continuellement de la matière organique à la place centrale que constitue leur nid. De plus, durant l'excavation et le nettoyage des nids, ils apportent du sous-sol aux surfaces des buttes sur lesquelles se trouvent leurs nids (Figure 5). Quant aux vers de terre, durant la saison des pluies ils concentrent leurs activités sur les buttes, où ils peuvent respirer, et leurs turricules sont donc aussi concentrées sur les buttes. La matière organique et minérale que tous ces organismes amènent aux buttes compense d'éventuelles pertes dues à l'érosion. Les ingénieurs ont aussi un autre type d'impact sur les buttes. Les structures biogéniques - les nids, les galeries, les agrégats résistants à l'eau - créées par les insectes sociaux, les vers de terre et les racines des plantes stabilisent le sol des buttes contre l'érosion. Les macropores créés par ces organismes favorisent l'infiltration de l'eau des pluies plutôt que son ruissellement, réduisant davantage le taux d'érosion. Ces organismes semblent donc avoir préservé l'héritage physique des activités humaines de 
construction de champs surélevés, longtemps après la disparition des ingénieurs humains (McKey et al. 2010 ; Renard et al. 2013).

Figure 5 : Dépôts sur le sommet d'un champ surélevé abandonné, par la colonie de la fourmi Acromyrmex octospinosus qui habite cette butte

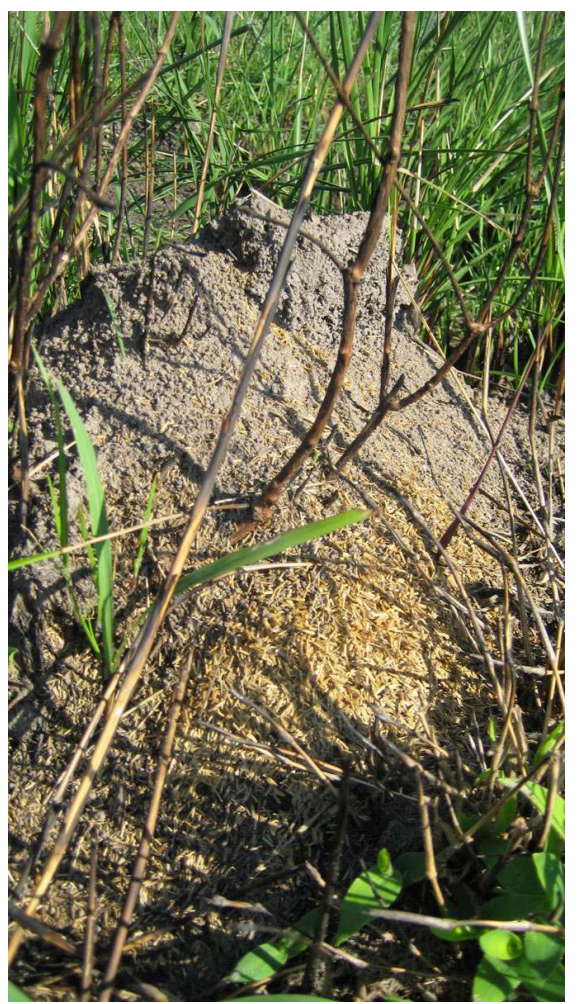

Matière minérale (sol gris au sommet, provenant de l'excavation du sous-sol) et organique (détritus végétal jaunâtre au premier plan : détritus végétal usé et rejeté de la chambre de culture de champignons des fourmis)

(c) D. McKey

Les paysages à champs surélevés précolombiens dans les savanes ${ }^{1}$ côtières de Guyane, paysages co-construits par l'Homme et la nature, n'ont suscité que peu d'intérêt en tant qu'objets de la conservation. Leur intérêt potentiel est virtuellement complètement ignoré, non seulement par les citoyens et les décideurs, mais également par la communauté des conservationnistes et gestionnaires d'aires protégées.

\section{Mais, les vestiges des systèmes agricoles abandonnés - par conséquent échoués- valent-ils réellement quelque chose?}

11 Les systèmes agricoles profondément ancrés dans le passé, mais toujours en activité aujourd'hui, offrent une preuve convaincante de durabilité. Ainsi, celui des chinampas (Merlin-Uribe et al. 2013), des terrasses andines (Sandor \& Furbee 1996), de la co-culture riz/poisson en Chine (Xie et al. 2011) et des terras pretas amazoniennes - qui sont encore créés et utilisés aujourd'hui (Schmidt 2013) - sont amplement acceptés comme des systèmes « ingénieux » et donc dignes de la conservation. Toutefois, argumenter qu'un système de culture soit ingénieux et durable est plus difficile quand il a aujourd'hui disparu. 
L'agriculture sur champs surélevés des basses terres sud-américaines en est un bon exemple. En effet, bien qu'encore largement pratiquée dans les tropiques du Vieux Monde (Denevan \& Turner 1974), notamment en Afrique (Pélissier 1966) et en Asie, cette technique agricole y est relativement peu étudiée, tandis qu'elle a fait l'objet de nombreuses recherches en Amérique du Sud où elle a pourtant quasiment disparue depuis la conquête européenne. La grande majorité de ces études a été faite par des archéologues et des géographes. Les premiers archéologues qui se sont intéressés à ces systèmes précolombiens, impressionnés par la grande étendue de vestiges de champs surélevés qu'ils découvrirent (Figure 6), considérèrent qu'ils avaient trouvé la preuve de denses populations humaines dans un environnement apparemment inadapté à l'agriculture intensive. Les efforts pour expliquer l'apparente haute productivité se basaient souvent sur l'hypothèse que ces systèmes fonctionnaient de manière similaire aux chinampas. Il faut en outre signaler que la disparition de ces systèmes agricoles de champs surélevés n'est en général apparemment pas liée à une faille technique mais à la baisse démographique et au remplacement de population dus à la conquête européenne.

Figure 6 : Étendue des complexes de champs surélevés dans la Savane de Corossony, à l'ouest de Sinnamary, Guyane française

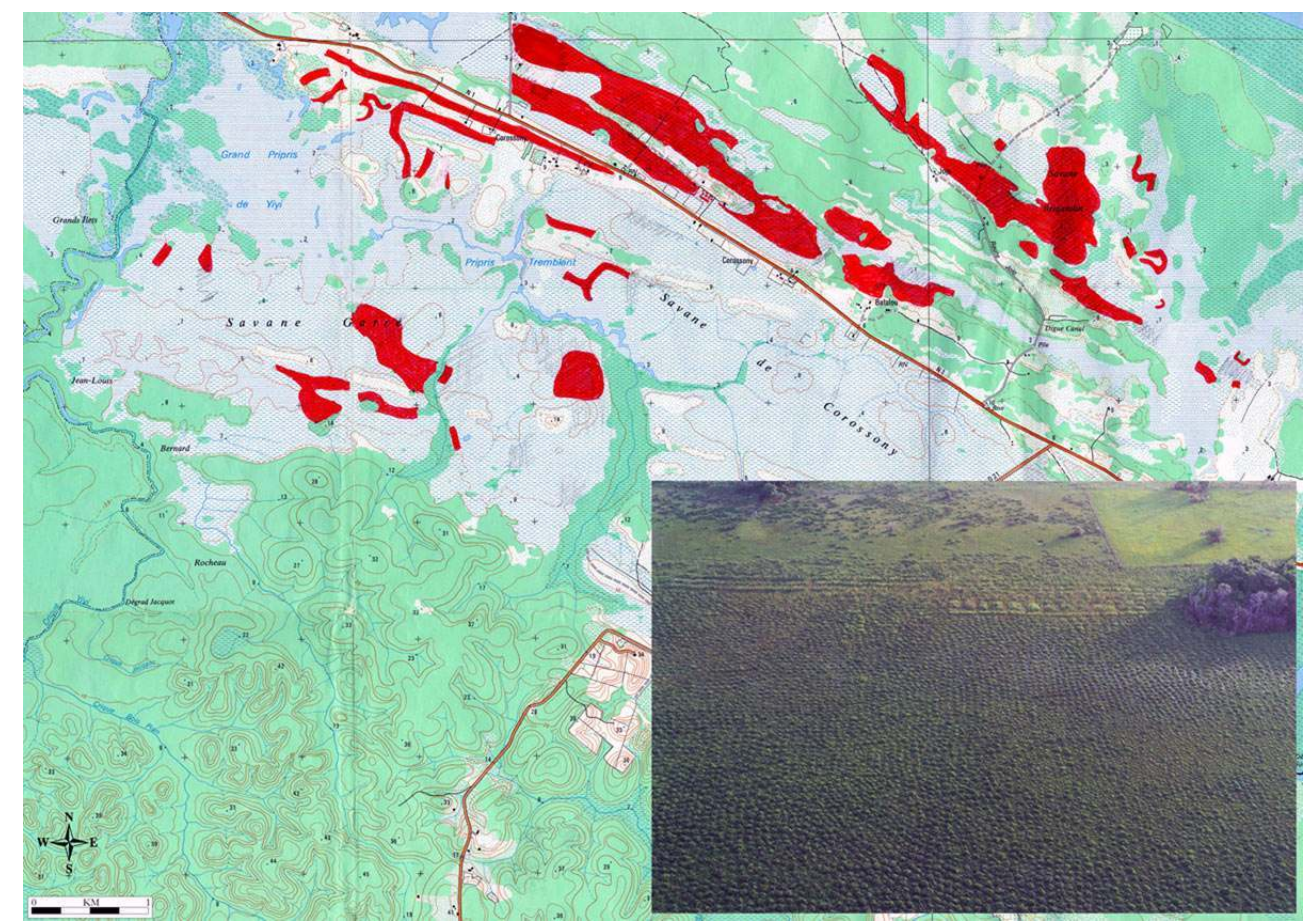

Photo et relevé S. Rostain sur fond de carte IGN

Toutefois, la supposition que l'agriculture sur champs surélevés était une sorte de «révolution verte précolombienne » est de plus en plus remise en question par certains (Lombardo et al. 2011). Malgré la multiplication d'études sur les champs surélevés précolombiens d'Amérique du Sud ces deux dernières décennies, l'intérêt sur le rôle qu'ils pourraient jouer dans le monde moderne a décliné. En revanche, le renouveau d'attention porté sur l'agriculture sur champs surélevés en Afrique, où elle perdure encore de nos jours, éclaire le rôle que de tels systèmes peuvent jouer (McKey et al. 2014). Bien que leur haute productivité soit encore mise en doute, il est évident que les champs surélevés démontrent une manière de faire coexister l'agriculture avec les terres humides et le 
maintien des qualités et de la biodiversité de leur écosystème. Si les avantages agronomiques de l'agriculture sur champs surélevés sont encore en débat (Renard et al. 2012; Baveye 2013), cette discussion a peu d'incidence sur la valeur des vestiges de champs surélevés comme patrimoine bioculturel.

\section{Menaces sur les vestiges de l'agriculture sur champs surélevés précolombiens dans les Guyanes}

\section{Inventaire des champs surélevés des Guyanes, leur état actuel et les menaces auxquels ils sont confrontés}

Il est vrai que l'agriculture sur brûlis domine aujourd'hui en Amazonie, mais on sait maintenant que des techniques agricoles plus intensives et permanentes furent mises en œuvre à l'époque précolombienne (Rostain 2008). Il est clair que peu d'entre elles ont laissé des restes monumentaux derrière elles. Néanmoins, la simple présence d'hommes a provoqué un impact dans l'environnement mais les anciennes modifications humaines du paysage varient en visibilité et signification.

L'étendue de ces remaniements est encore grandement sous-estimée par certains, qui considèrent que seuls les colonisateurs occidentaux furent enclins à tenter, et capables d'effectuer, de grands remaniements de paysages (Figure 6) : «Ces populations [indigènes] entretenaient des rapports symbiotiques avec leur milieu, car leurs prélèvements ne dépassaient jamais la capacité naturelle de régénération des écosystèmes; la chasse, la pêche et la cueillette constituaient alors leur principale source de subsistance. C'est l'arrivée des premiers colons qui perturba le système » (Transler et al. 2006: 70). Tandis que l'impact des communautés indigènes précolombiennes est de plus en plus reconnu ailleurs en Amérique du Sud (Mann 2005 ; Balée \& Erickson 2006), cet impact est souvent complètement ignoré sur le littoral guyanais, et seul les effets des populations occidentales sont considérés, qu'ils soient négatifs ou positifs. Et pourtant, il a été aujourd'hui bien démontré que l'influence amérindienne sur le paysage débuta il y a quelques milliers d'années et fut intensifiée peu avant le début de l'Ère Chrétienne.

Si les premiers champs surélevés de la région ont été datés des environs de 300 apr. J.-C. à l'ouest du Suriname par association avec des tertres résidentiels voisins, ils remontent en majorité de l'époque Arauquinoïde (600-1500 apr. J.-C.). Les datations les plus précises ont été faites durant notre projet à l'aide de matières organiques provenant de paléosols enterrés sous les buttes de deux complexes différents des alentours de Kourou. Les âges au ${ }^{14} \mathrm{C}$ obtenus sont respectivement de $760+/-40$ ans AP (calibré 670-700 ans AP) et 1010 +/- 40 ans AP (calibré 920-950 ans AP) (McKey et al. 2010). Ces datations correspondent très exactement à celles obtenues durant nos fouilles dans les proches sites d'habitat de culture Arauquinoïde. Les plus anciens champs surélevés de Guyane française furent donc construits au moins à la fin du premier millénaire de notre ère, si ce n'est avant, et vraisemblablement utilisés jusqu'aux débuts de la conquête européenne, au XVI ${ }^{\mathrm{e}}$ siècle. On ne peut rester que stupéfait devant leur excellent état de conservation plus de 500 ans après, même en prenant en compte l'action préservatrice des organismes naturels 'ingénieurs du sol' présentés plus haut.

Bien plus, les aires impliquées par les structures monumentales précolombiennes sur la côte des Guyanes sont bien plus grandes que celles modifiées par les colons depuis le XVII ${ }^{\mathrm{e}}$ 
siècle, quand ils commencèrent à construire des polders. Pour la seule Guyane française, l'inventaire précis réalisé par l'un des auteurs sur la base d'interprétations stéréoscopiques de milliers de clichés aériens a permis de calculer une superficie de près de 3000 hectares transformée par les terrassiers précolombiens, sachant qu'une bonne partie des structures anciennes n'a pu être vue car aujourd'hui disparue pour diverses raisons (Rostain 2012a).

Il faut préciser ici que les terrassements précolombiens ne se limitent pas aux champs surélevés, mais comprennent également les canaux, les réservoirs, les dérivements ou redressements de cours d'eau, les digues, les chemins surélevés et les tertres d'habitat ou cérémoniels. Les perturbations de l'écosystème dues à ces constructions ont altéré de façon permanente le milieu. Après la construction, la mise en culture puis l'abandon des champs surélevés, les savanes littorales guyanaises ne retrouvèrent jamais leur dynamique originelle ; l'intervention humaine a provoqué une hétérogénéité pérenne qui fut ensuite maintenue par les ingénieurs d'écosystème naturels (McKey et al. 2010).

La transformation intensive du paysage précolombien avait évidemment un coût humain, justifié par les bénéfices à long terme que ces innovations apportaient (Rostain 2012a). En édifiant des milliers de champs surélevés, des chemins surélevés et des tertres d'habitat et en creusant des canaux et des réservoirs, les Amérindiens investissaient un territoire afin de l'adapter à leurs besoins pour plusieurs siècles. Il en résulte que partout où ont habité les Amérindiens, le milieu a été plus ou moins transformé, volontairement ou pas. Les modifications majeures ont été faites en terre, que ce soit par creusement ou par édification.

Jusqu'à présent, les scientifiques se sont focalisés sur les plaines fluviatiles des bassins de l'Amazone et de l'Orénoque, aussi fertiles pour les plantes que pour les sociétés humaines, oubliant un peu d'autres espaces tout aussi prolifiques. La várzea ne fut pas l'unique terreau des populations stratifiées d'Amazonie, car la façade Atlantique, les grandes savanes (llanos) vénézuéliennes et boliviennes, tout comme le piémont volcanique des Andes, connurent des développements de sociétés complexes bien plus denses que dans l'intérieur forestier et qui ont organisé des terrassements notables, encore visibles aujourd'hui (Rostain 1991, 2010, 2012b ; Spencer et al. 1994 ; Erickson 2003 ; Walker 2004). Dès lors, il est impossible d'accepter, d'une part, l'existence d'une mosaïque d'environnements distincts en Amazonie et, d'autre part, de rejeter la possibilité d'un éventail diversifié de cultures humaines coexistant depuis l'époque précolombienne. Il faut prendre en compte les subtiles variations d'un groupe à l'autre, parfois encore visibles aujourd'hui, et qui furent très vraisemblablement encore plus marquées avant la conquête européenne.

21 Par ailleurs, les chercheurs ne peuvent raisonnablement espérer retrouver tous les vestiges de terrassements abandonnés car beaucoup ont été dégradés ou détruits au cours du temps. Par exemple, une grande partie des champs surélevés de Savane Maillard, en Guyane française, ont été complètement anéantis, sans compter les alignements euxmêmes, qui ont quelquefois été en partie effacés car les buttes se sont réduites à des mottes irrégulières et sans forme (Figure 7). Similairement, de nombreux canaux ont été partiellement ou complètement remplis par des colluvions de sédiments. 
Figure 7 : Champs surélevés érodés après de multiples incendies, Savane Maillard, Guyane

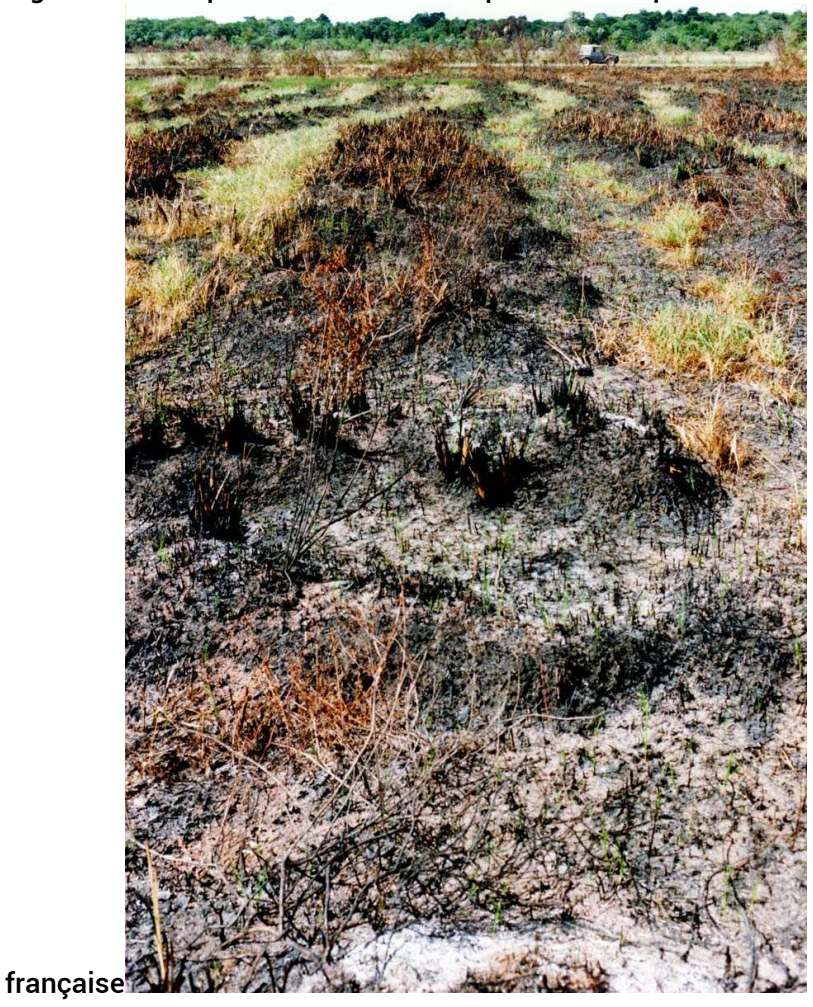

(c) S. Rostain

L'étude des terrassements précolombiens est particulièrement difficile en Amazonie, où des structures plus récentes ont fréquemment été construites sur les plus anciennes. Bien plus, des formations naturelles peuvent aisément être confondues avec des constructions humaines, qui ont peut-être été influencées, à l'origine, par l'observation du paysage naturel (McKey et al. 2014). En outre, sans étude et datation archéologique - cette dernière étant parfois impossible à réaliser - il est souvent difficile de vérifier la réelle antiquité des structures.

Comme il a été signalé auparavant, les canaux creusés par l'homme sont particulièrement compliqués à identifier et confirmer. Les Amérindiens, mais également les colons, ont dans certains cas dévié, redressé ou prolongé des ruisseaux et des rivières (Figure 8). Plusieurs exemples viennent à l'esprit, tels les fréquents fossés de ceinture qui contrôlent la hauteur de l'eau dans les complexes de champs surélevés des Guyanes ou les réseaux de canaux qui empêchent les inondations autour des monticules d'habitat du Suriname (Figure 9). 
Figure 8 : Creusement d'un canal de dérivation par des orpailleurs en Guyane française

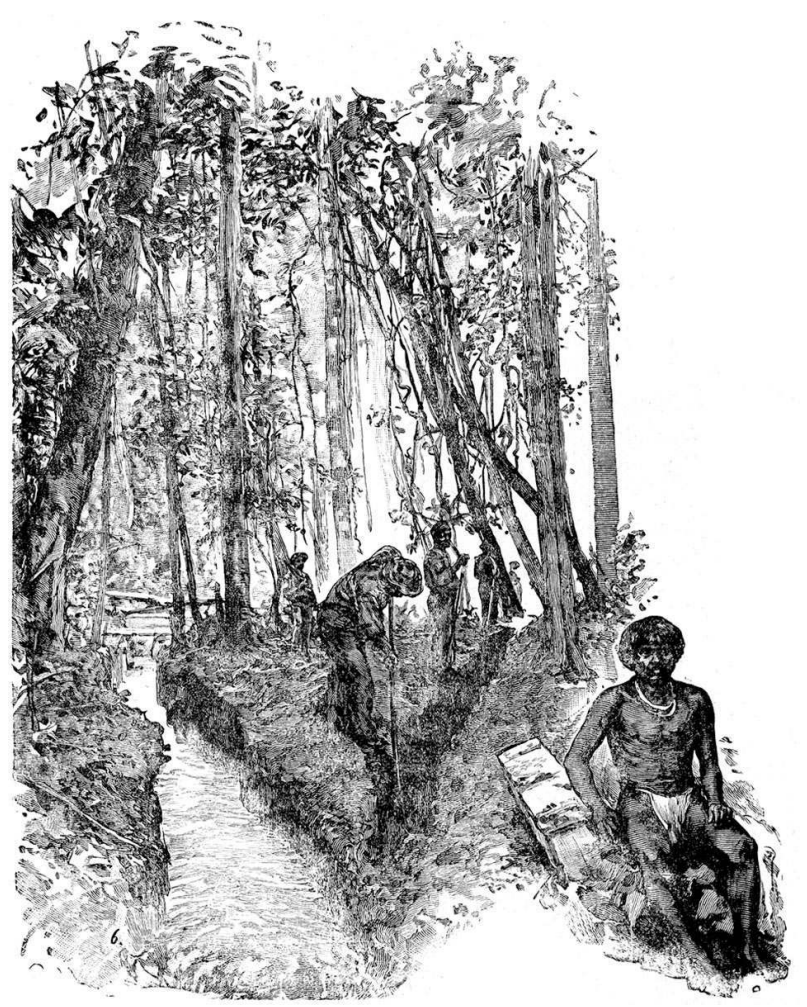

In Villars $1914: 133$

Figure 9 : Réseau de canaux autour du tertre artificiel de Wageningen-3, côte occidentale du Suriname

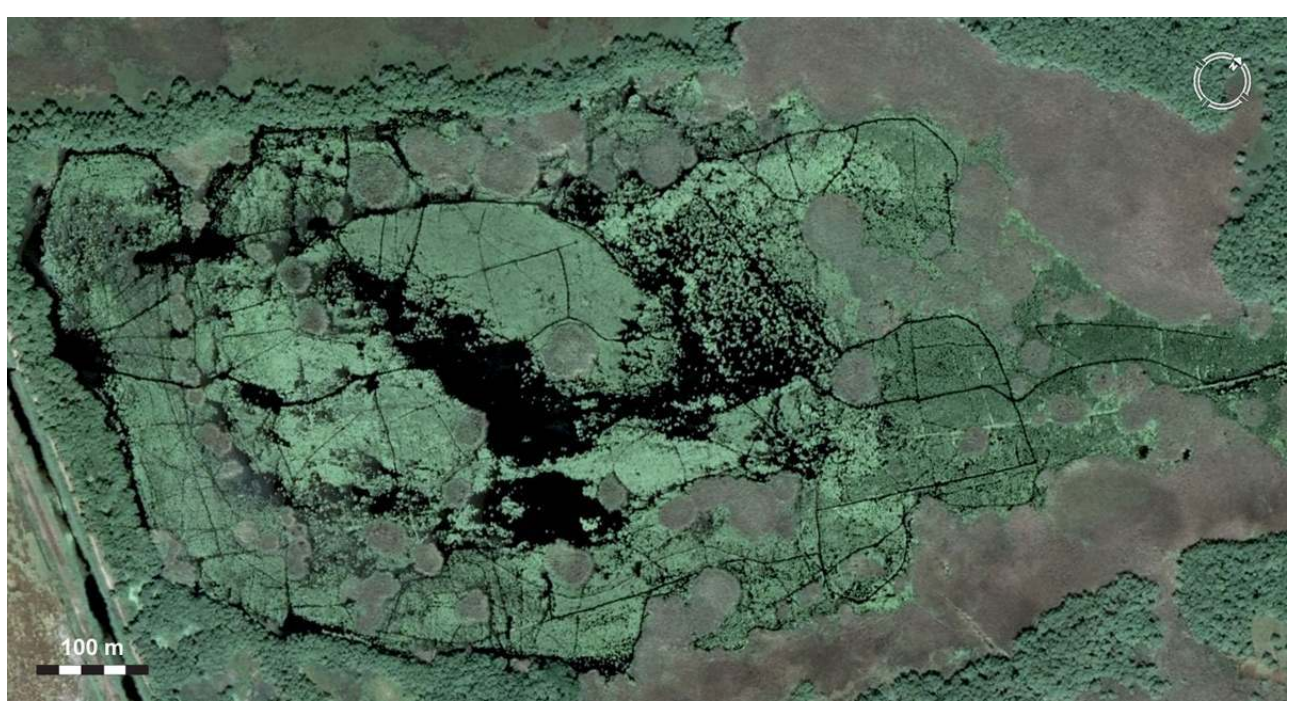

(c) Google Earth

24 Les structures précolombiennes de ces paysages peuvent être effacées par différents processus naturels, mais ce sont bien les mécanismes d'alluvionnement et de colluvionnement, souvent liés à des remaniements anthropiques récents, qui sont les plus proéminents pour expliquer les phénomènes actuels d'immersion totale de certaines structures ou, au contraire, de colonisation de la forêt. La raison de la submersion 
actuelle de certains champs surélevés, dans des aires particulièrement inondables, réside souvent dans leur abandon. Quoique les phénomènes d'érosion soient relativement peu importants dans la plaine côtière ancienne de Guyane, il existe toutefois des modifications de la surface du sol, en grande partie provoquées par les précipitations. Ainsi, les buttes précolombiennes peuvent être attaquées par ce type d'érosion, qui déstructure les champs surélevés pour les rendre irréguliers et éclatés en mottes. Le cas le plus spectaculaire a été observé dans la Savane Maillard, au sud de Macouria, où les monticules eux-mêmes se sont divisés en deux ou quatre micro-buttes, voire davantage. Il s'agissait à l'origine d'alignements de buttes carrées, de $280 \mathrm{~cm}$ de côté en moyenne, et séparées par des canaux d'environ $70 \mathrm{~cm}$ de largeur (Figure 7).

Cette érosion est accrue par les feux de savane annuels qui suppriment le couvert végétal, et donc la protection contre l'érosion. Un sondage pédologique au pied d'une butte a montré le processus d'aplanissement général des champs surélevés : la terre de la butte tendant à glisser dans les canaux intermédiaires, reliefs et canaux s'effacent. Dans certains cas, il ne reste quasiment plus de trace morphologique du champ surélevé mais, parfois, la végétation conserve quand même l'empreinte du bas-fond humide et de la butte plus sèche, par l'alignement d'espèces végétales différentes entre ces deux habitats (Figure 10). L'entretien des champs surélevés devait nécessiter de régulièrement curer les canaux et remonter les matériaux sur le sommet des buttes. Dès l'abandon de la mise en culture, s'est amorcé un lent aplanissement, éventuellement accéléré par les interventions anthropiques récentes (mise à feu des savanes, constructions) déjà mentionnées.

Figure 10 : Lignes de graminées et cypéracées marquant l'existence passée de champs surélevés, aujourd'hui totalement aplanis et invisibles dans le modelé

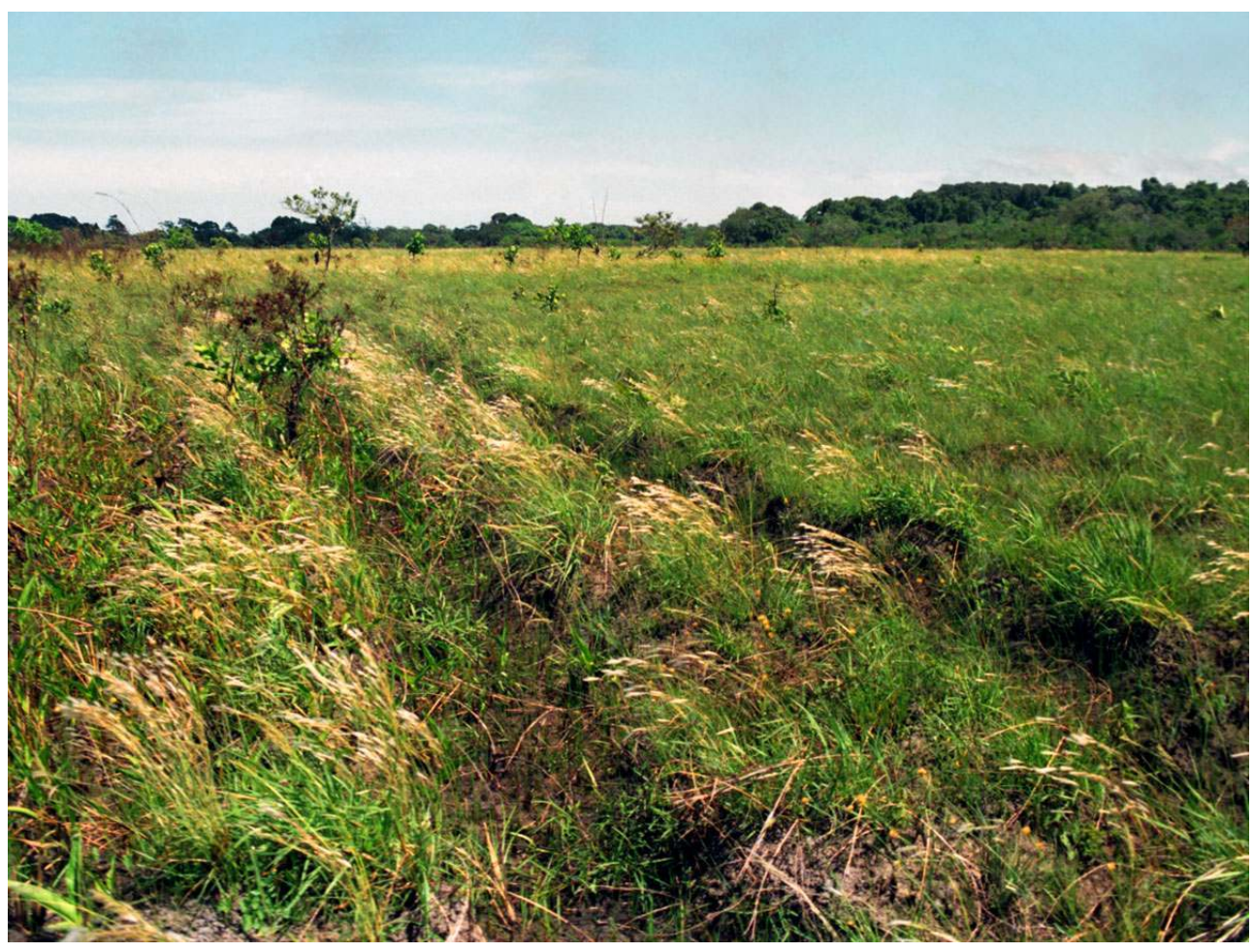

(c) S. Rostain 
À ces facteurs principaux que sont l'érosion et l'aplanissement, on peut rajouter une autre cause naturelle de destruction propre à la région. La côte des Guyanes présente en effet une évolution extrêmement dynamique avec des phases de progradation et d'autres, moins conséquentes, de recul du rivage. En effet, le tracé de la côte de Guyane changeant fréquemment et rapidement, sa situation actuelle est très différente de celle de l'époque précolombienne. La ligne de rivage se modifie très rapidement en fonction de phases d'accumulation et d'érosion. Des bancs de vase, apportés par le système de dispersion amazonien, transitent le long de la côte en laissant des espaces inter-bancs, généralement constitués de plages sableuses. Si l'ouest de Kourou présente aujourd'hui une belle plage, un énorme banc de boue recouvert de mangrove l'occupait il y a 25 ans. Cette dynamique de la côte est à l'origine de la destruction fréquente de sites littoraux. Par ailleurs, des sites résidentiels ou de champs surélevés autrefois localisés sur le rivage peuvent se retrouver beaucoup plus à l'intérieur de la plaine côtière et, à l'inverse, certains sites pouvaient être plus éloignés de la mer à l'époque de leur occupation. Bien que les possibilités aient probablement été rares durant le dernier millénaire depuis l'abandon des structures de terre, on ne peut écarter complètement le risque de disparition complète de savanes sous la mer lors d'un épisode de recul du rivage (Figure 11).

Figure 11 : Évolution rapide du rivage guyanais : le cas de la savane de Grand Macoua
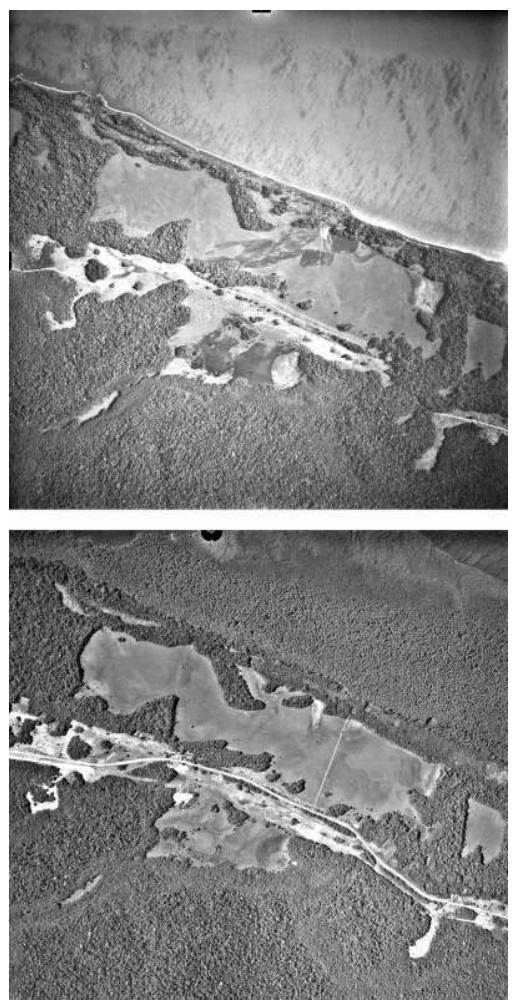

En 1976, l'océan vient butter directement au pied du cordon sableux (en haut) et, en 2001, une large bande de mangrove a colonisé le front de mer qui s'est déplacé vers le nord (c) IGN

Outre ces mécanismes naturels, il faut prendre en compte les activités humaines. Depuis l'époque coloniale et continuant aujourd'hui, le feu, l'agriculture et l'élevage, les terrassements, et les travaux sur les cordons élevés associés aux zones humides accélèrent la destruction des structures de terre précolombiennes. C'est cette menace 
d'origine anthropique, avec souvent une destruction directe par des travaux modernes, qui est la plus à craindre. Ainsi, les dangers comprennent des interventions actives, tels des drainages, des labours, des travaux immobiliers ou des conversions en champs de riz. Bien plus, les sites domestiques archéologiques associés aux champs surélevés, sont bien davantage en danger. Ils sont généralement localisés sur les rares aires bien drainées, de longs et étroits cordons sableux appelés cheniers, qui courent parallèlement au rivage à intervalles irréguliers, mesurent plusieurs dizaines de mètres de large et dépassent parfois une centaine de kilomètres de long. La construction de la Route Nationale 1, et les installations associées (résidentielles, industrielles, etc.), ont oblitéré des dizaines de kilomètres de ces cordons, tandis que d'autres ont été réduits à néant par les nombreuses carrières de sable.

\section{Le feu}

L'incendie est vraisemblablement le plus ancien et le plus dangereux ennemi des terrassements précolombiens des savanes côtières des Guyanes. En effet, à l'instar de la plupart des savanes des Néotropiques, les basses terres littorales des Guyanes sont sujettes à de nombreux incendies annuels d'extension très variable. L'origine de ces feux est incertaine, bien qu'il semble que si certains peuvent être naturels, ils sont pour l'essentiel le fait de l'Homme (Figure 12). Hélas, les enquêtes ethnologiques menées auprès des populations locales n'éclaircissent pas vraiment les raisons de ces embrasements car les réponses sont aussi diverses que les personnes interrogées: assainissement du sol, destruction des plantes et animaux nuisibles, accident, jeu, etc. Il a pu en revanche être démontré qu'avant la conquête européenne, durant la période de mise en culture des champs surélevés, les agriculteurs amérindiens contrôlaient et empêchaient les feux de savanes (Iriarte et al. 2012). 
Figure 12 : Feu anthropique dans un complexe de champs surélevés de la côte centrale de Guyane française

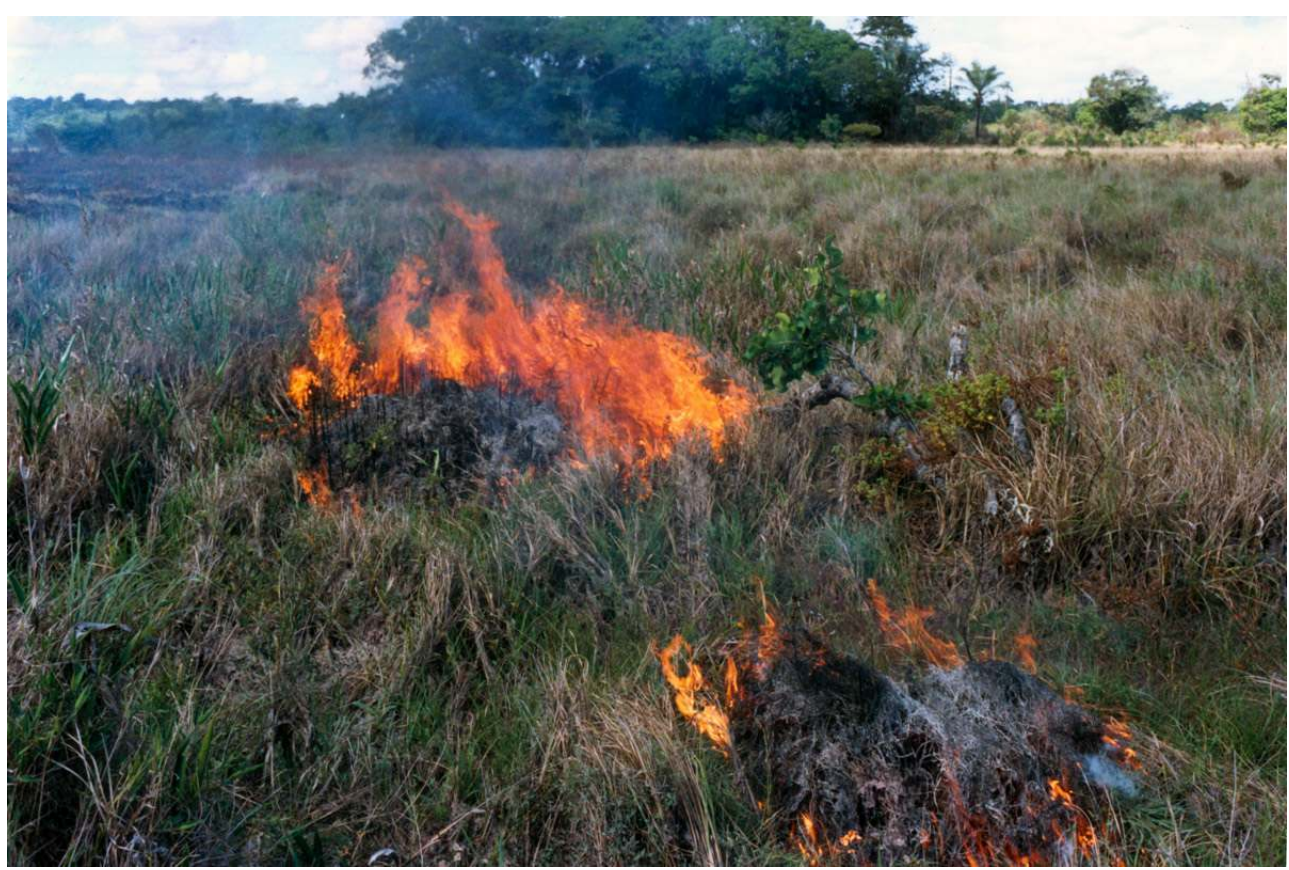

Le sommet des buttes, plus touffu et sec, brûle avant les bas-fonds gorgés d'eau

(c) S. Rostain

Quoi qu'il en soit l'impact des incendies sur les savanes littorales n'est pas négligeable, les superficies détruites, très variables d'une année à l'autre, se chiffrant à plusieurs hectares sur l'ensemble du territoire. Les plantes sont les premières atteintes et, même si les graminées et les cypéracées ont repoussé l'année suivante, certaines espèces sont irrémédiablement détruites. Par exemple, un village Palikur abandonné, localisé en savane, fit l'objet d'une étude archéologique par l'un des auteurs en 1989 durant laquelle un inventaire exhaustif des plantes cultivées fut dressé (Rostain, en cours de publication). Vingt ans plus tard et après plusieurs incendies, il ne restait aucune des espèces introduites par les anciens habitants des lieux.

Les conséquences des feux de savane sont encore plus importantes sur les champs surélevés car ils les privent temporairement du couvert végétal qui les protège de la forte érosion pluviométrique qu'ils subissent, la moyenne annuelle des précipitations variant autour de $3000 \mathrm{~mm}$ (Figure 13). La déstructuration des buttes est fortement accélérée face à un tel phénomène qui a pu être en partie mis en évidence grâce aux études pédologiques. Par exemple, dans la savane Grand Macoua les analyses de répartition de Césium $137\left({ }^{137} \mathrm{Cs}\right)$, déposés sur la surface de la terre lors des essais nucléaires des années 1950 et 1960, démontrent clairement une lente érosion de sol, qui est déposé dans les parties basses (Pfahler et al. 2015). Les buttes persistent grâce au maintien, dans cette savane, des organismes ingénieurs qui amènent continuellement de la matière organique et minérale au sommet des buttes (McKey et al. 2010), comme expliqué ci-dessus dans la première section. 
Figure 13 : Champs surélevés érodés et repousse de la végétation après un incendie, Savane Matiti, Guyane française

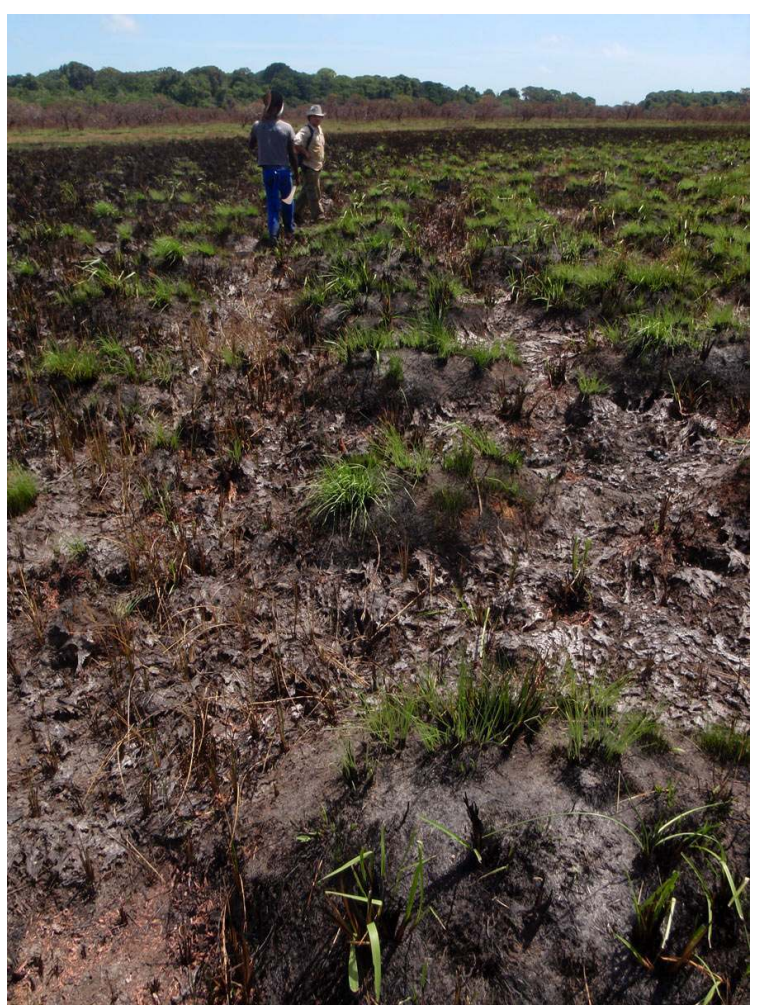

(c) S. Rostain

\section{Agriculture et élevage}

31 L'utilisation moderne des savanes littorales, que ce soit pour les cultiver, y faire paittre le bétail ou autre activité paysanne, peut être très destructive. Ainsi, les labours et les piscines à poissons altèrent complètement la surface de la savane, effaçant par là même les monticules anciens et les drainages antérieurs (Figure 14). 
Figure 14 : Prairie aplanie mécaniquement par une pelleteuse ayant effacé des champs surélevés précolombiens près de Macouria, Guyane française

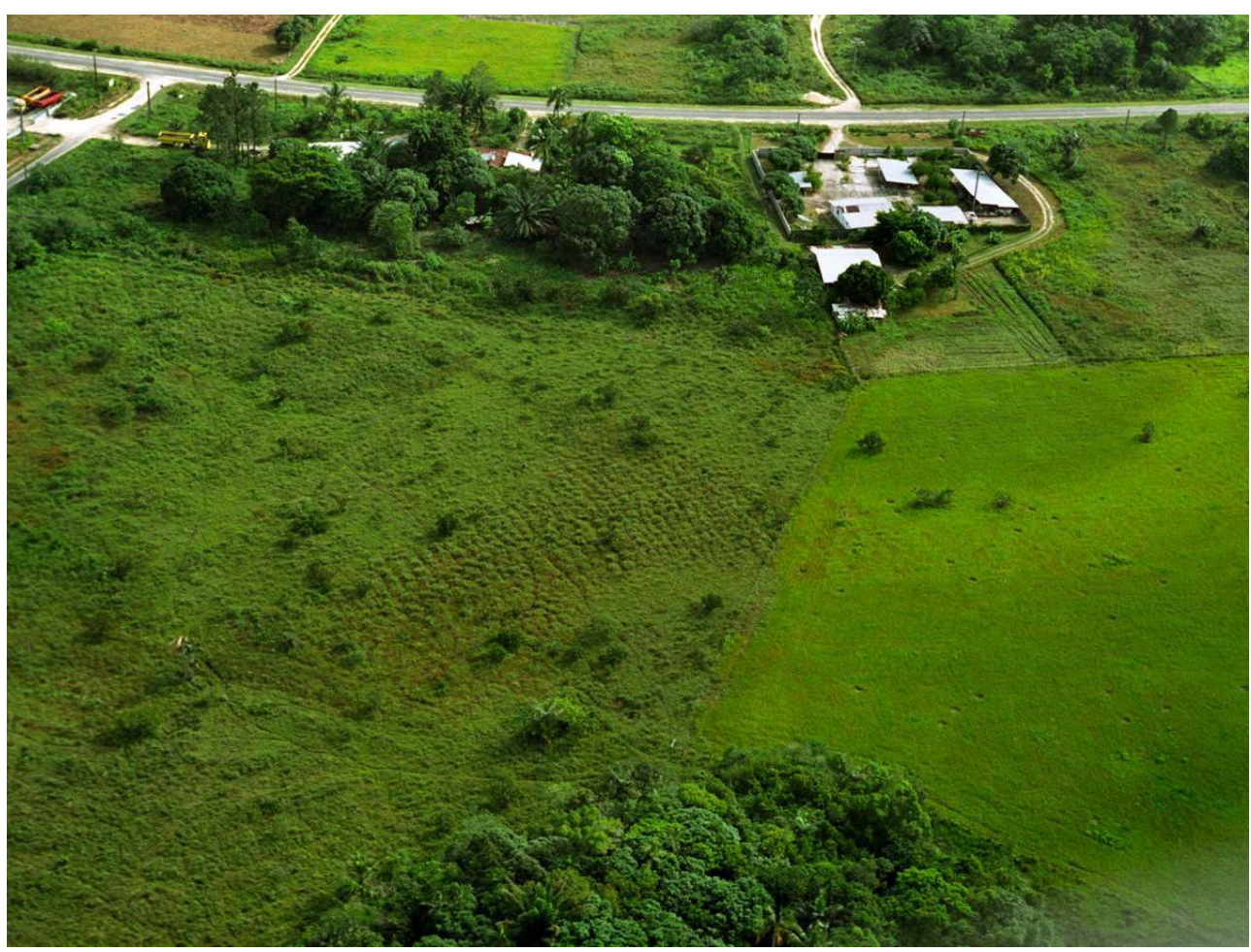

(c) S. Rostain

32 Toutefois, les premiers migrants européens ont réalisé des modifications encore plus profondes dans le paysage en construisant des polders étendus, parcelles à l'origine inondées que l'on a entourées de digues pour les assécher (Figure 15). En effet, durant plus de deux siècles, les Hollandais construisirent des polders sur des milliers d'hectares au Suriname, puis les Anglais, marchant sur leurs brisées, au Guyana. S'il n'y avait que 23 plantations au Suriname en 1668, elles étaient 641 en activité en 1800 le long du fleuve Para et dans les estuaires des fleuves Suriname et Commewijne (The National Planning Office of Suriname \& Regional Development and Physical Planning Department 1988). Il en résulte que la plaine côtière du Guyana et du Suriname est presque entièrement recouverte de polders (Figure 16). La canne à sucre était la principale culture, transportée sur des barges le long des canaux jusqu'à une sucrerie pour y être transformée. De nos jours, au contraire, les rares polders actifs produisent essentiellement du riz. En Guyane française, d'immenses polders furent ainsi aménagés dans les marais du bas Mana en 1986 pour la culture intensive du riz. 
Figure 15 : Polder colonial à l'est du Suriname

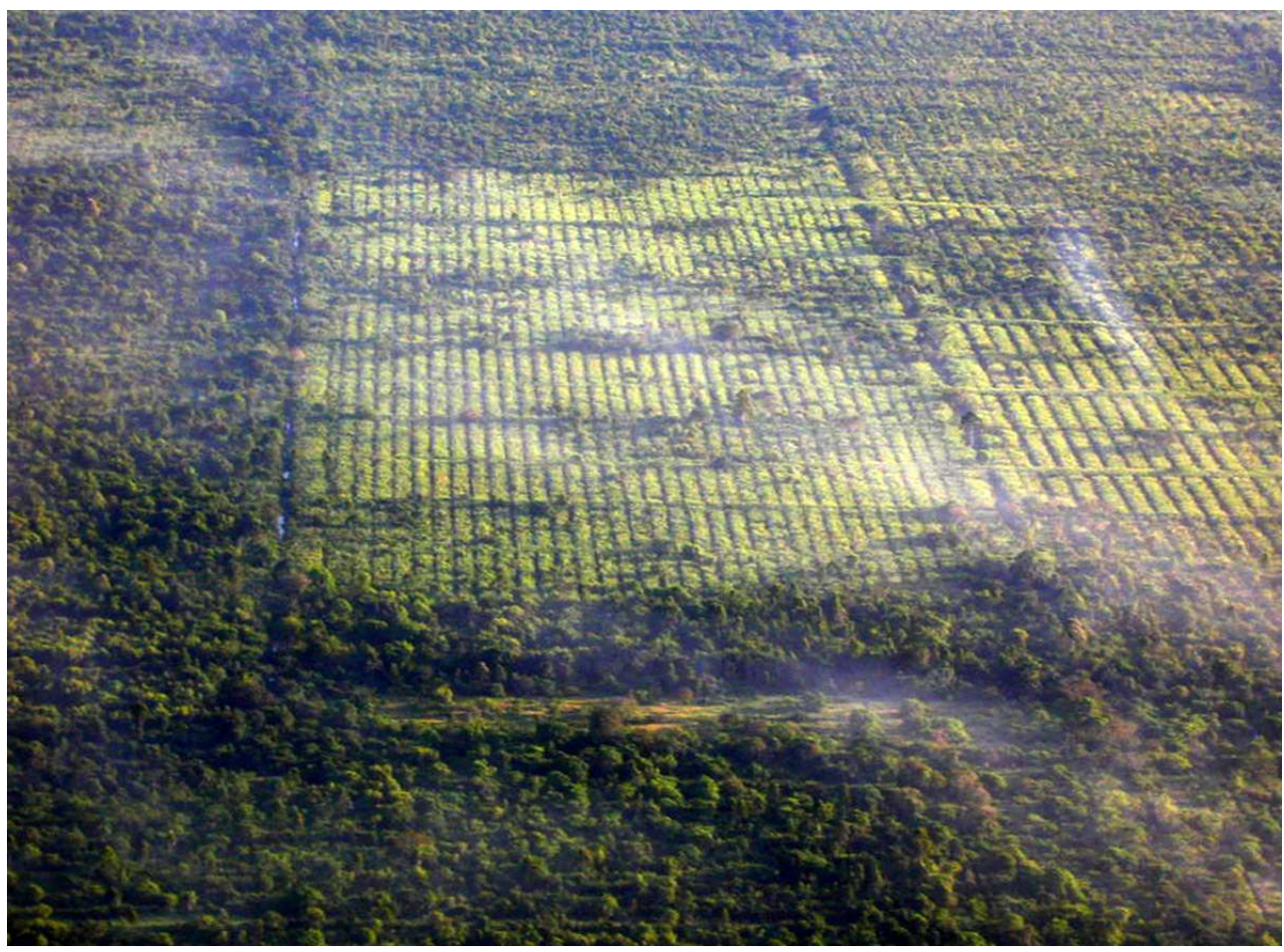

(c) S. Rostain

Figure 16 : Anciens polders abandonnés près du rivage, à l'est du Suriname

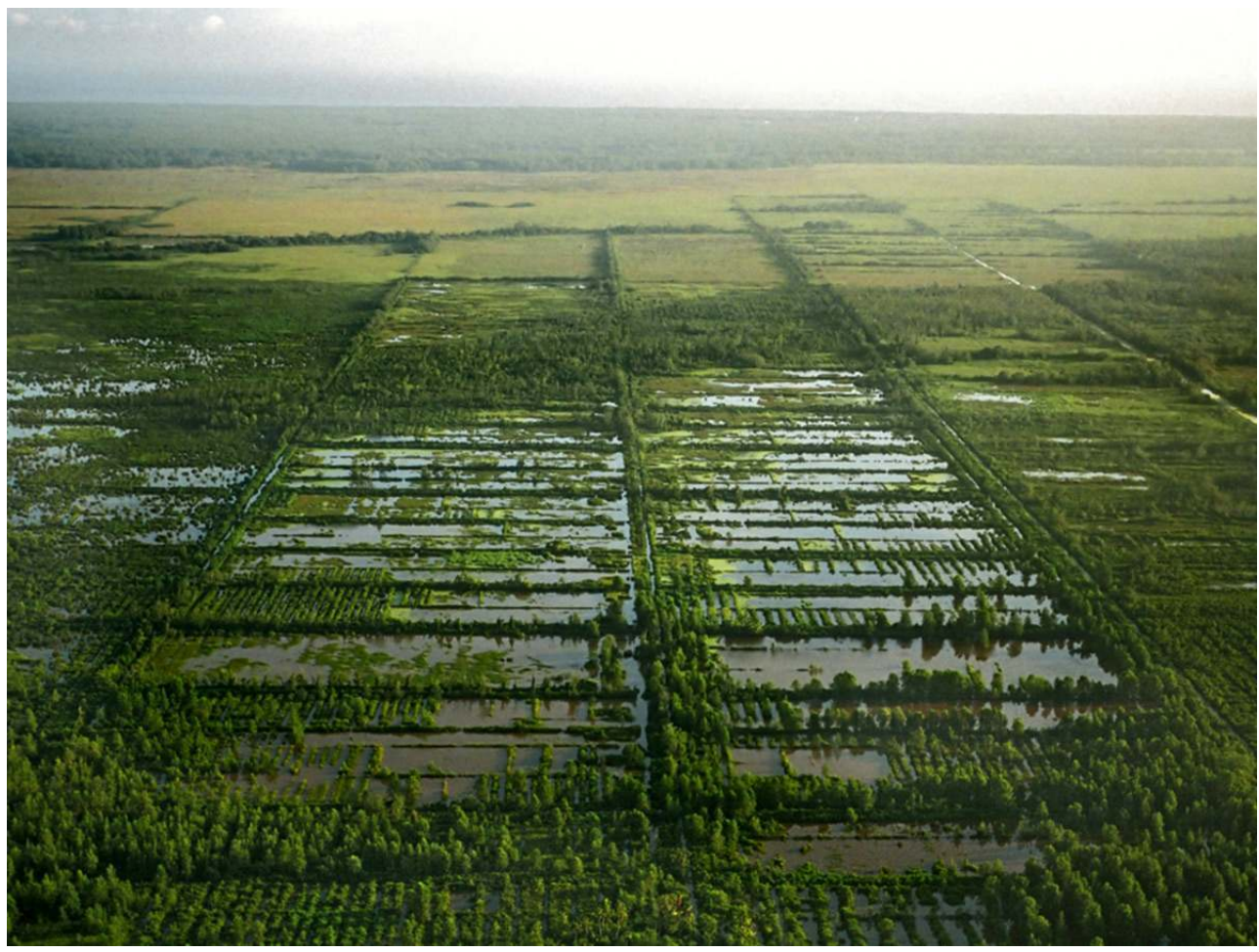

(c) S. Rostain 
Parallèlement, de la fin du XVIII à la fin du XIX ${ }^{e}$ siècle, très peu de polders furent édifiés en Guyane française, et la plupart à l'est de l'île de Cayenne. Cette réticence à la construction de ces parcelles agricoles en Guyane française a un résultat aujourd'hui bénéfique pour l'archéologie si l'on évalue les restes de champs surélevés existant dans les trois Guyanes, ceux du Guyana et du Suriname ayant été presque totalement effacés par les polders coloniaux. Ainsi, des milliers de champs surélevés ont été arasés ou enterrés par ces polders, rendant malheureusement impossible l'estimation de leur extension originelle. Notons qu'exceptionnellement, des vestiges de champs surélevés peuvent être discernés sous les polders lors de survols aériens (Figure 17).

Figure 17 : Vestiges de champs surélevés encore visibles dans le coin d'un polder moderne, à l'ouest du littoral de Guyane française

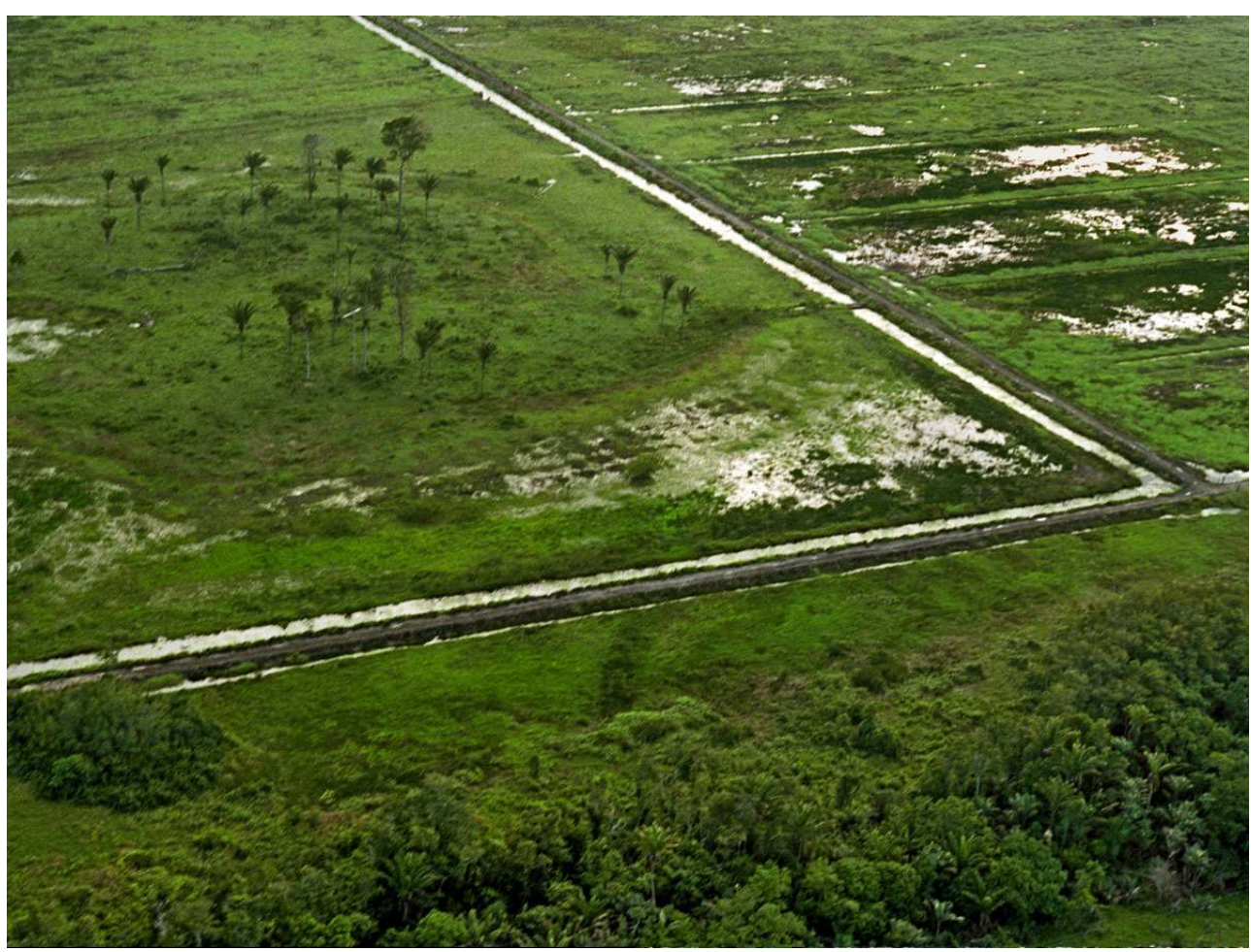

(c) S. Rostain

\section{Terrassements modernes}

34 Une autre menace importante sur ces paysages est l'aménagement de certaines aires de la plaine côtière depuis l'époque de construction des champs surélevés. Ce danger s'est évidemment amplifié au $\mathrm{xx}^{\mathrm{e}}$ siècle avec l'accroissement progressif de population vivant sur la frange littorale - en Guyane française, plus de 70 \% de la population vit sur la côte qui ne représente pourtant que $5 \%$ du département -, phénomène qui s'est fortement accéléré ces dernières années sous la pression de nouveaux migrants.

Le secteur nord de la Montagne des Pères, près de Kourou, est un bon exemple car il montre d'évidentes différences entre les photographies aériennes de 1955 et de 1987. En 1955, plusieurs alignements de petits tertres, tout comme des fossés de ceinture et une levée de terre, apparaissent clairement dans le marais, le long des cordons sableux plus élevés. En 1987, les mêmes buttes sont presque invisibles, tandis que les fossés de ceinture 
et la digue ont complètement disparu à cause d'un excès de sédimentation et d'érosion. Un survol de cette aire en ULM en 1989 et des prospections terrestres en 2008 ont confirmé la destruction de ces terrassements (Figure 18). Si les champs surélevés se sont conservés pendant plusieurs siècles, une brusque disparition de certains d'entre eux en une trentaine d'années peut sembler étonnante. L'explication réside dans les récents aménagements publics : il y a quelques années, des travaux dévièrent la Route Nationale 1 au sud de son tracé initial et, afin de relier l'ancienne et la nouvelle route nationale, un chemin nord-sud fut construit par apport de terre, qui barra transversalement les marais entre les cheniers. Ce chemin a dû influer considérablement sur le réseau hydrographique de cette aire, provoquant de nouvelles retenues d'eau et des apports de matériaux, peutêtre des alluvionnements ou des colluvionnements, qui auraient progressivement effacé les buttes. Un phénomène similaire d'effacement a été observé à la sortie de Kourou, là encore au niveau d'une déviation de l'ancienne route nationale. Nombreuses sont ainsi les routes modernes, parfois surélevées, coupant les complexes de champs surélevés (Figure 19).

Figure 18 : Disparition de structures précolombiennes dans le secteur nord de la Montagne des Pères, près de Kourou

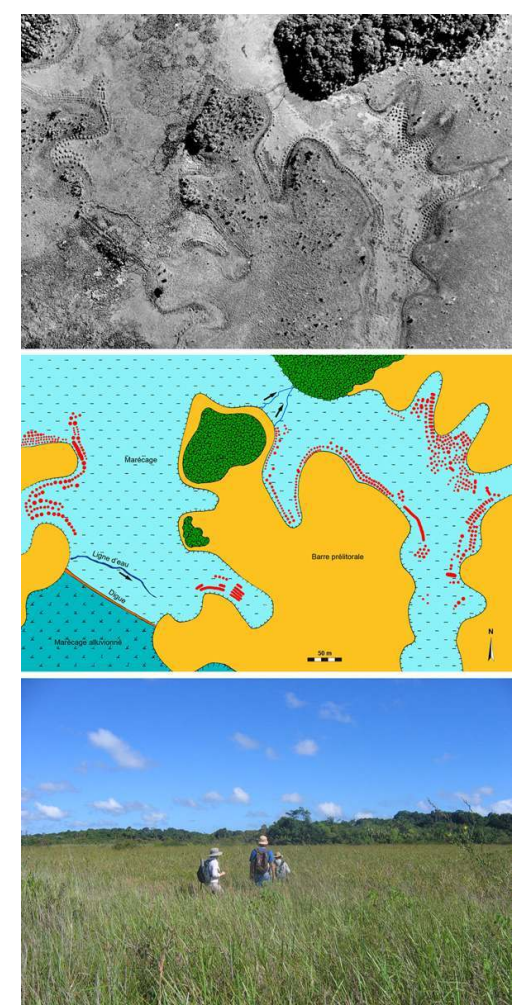

Entre 1955 et 2001, on ne retrouve plus la digue, ni plusieurs champs surélevés Photo et dessin S. Rostain, IGN 
Figure 19 : Route moderne traversant un complexe de champs surélevés dans la Savane Maillard, Guyane française

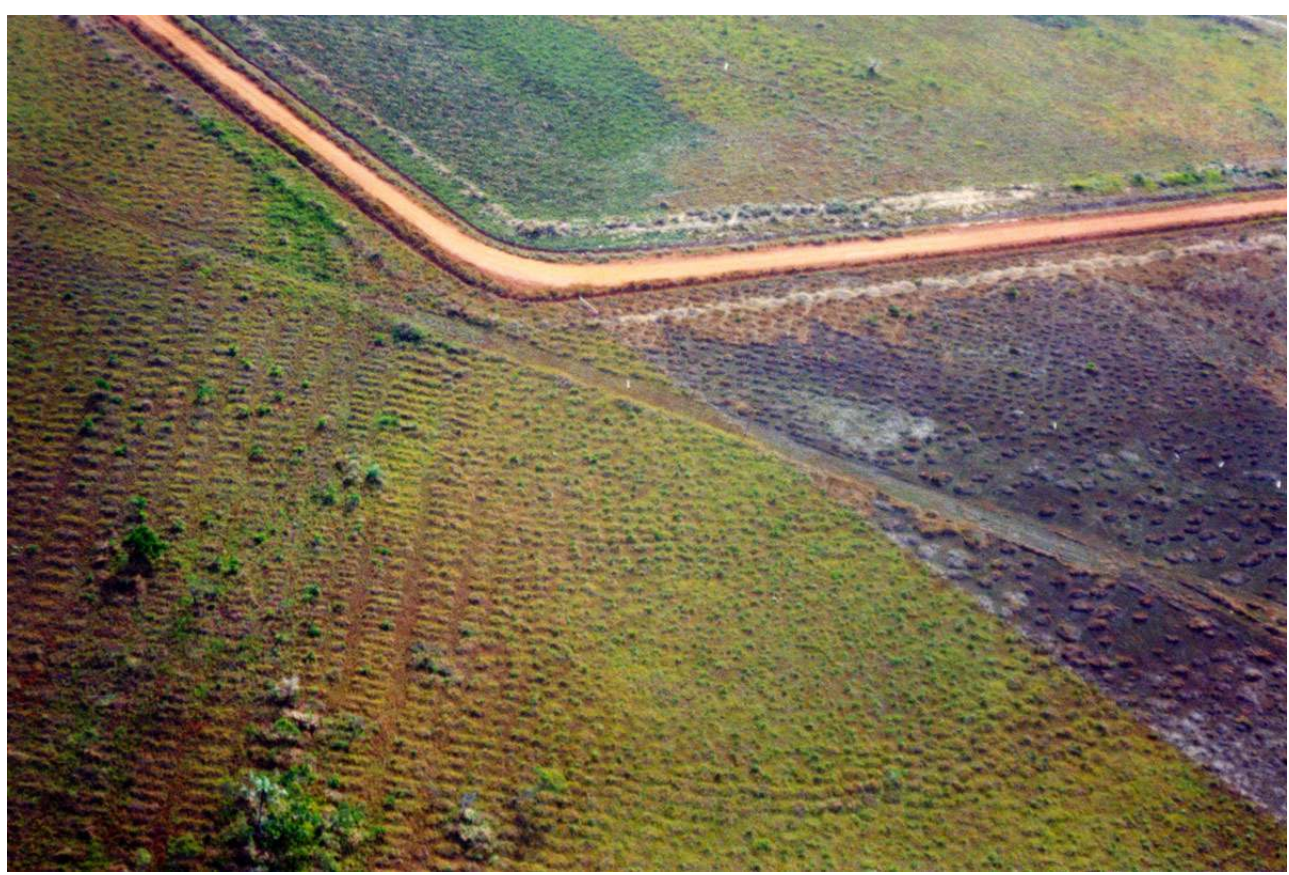

(c) S. Rostain occidentale récente de Suriname, vers 1964, modifia les conditions hydrographiques. L'absence de cheniers dans cette région nécessita l'édification d'une petite digue pour supporter la route. Après quelques années, les canalisations de drainage s'étaient bouchées et l'eau ne circulait plus entre le nord et le sud. Ne subissant plus les effets de marée, le marécage méridional se transforma en marais d'eau douce exclusivement. Au nord, où l'eau douce ne parvenait plus, les conditions devinrent plus salées et les sédiments, qui ne s'évacuaient plus, tendirent alors à occulter progressivement plusieurs groupes de champs surélevés. Une comparaison entre des photographies aériennes de 1947 et des clichés récents montre clairement ce phénomène d'effacement des structures.

Les constructions immobilières peuvent également avoir des effets néfastes similaires, provoquant le colluvionnement des buttes par le changement des conditions hydrographiques (Figure 20). 
Figure 20 : Champs surélevés en partie colluvionnés à cause d'une construction immobilière près de Mana, Guyane française

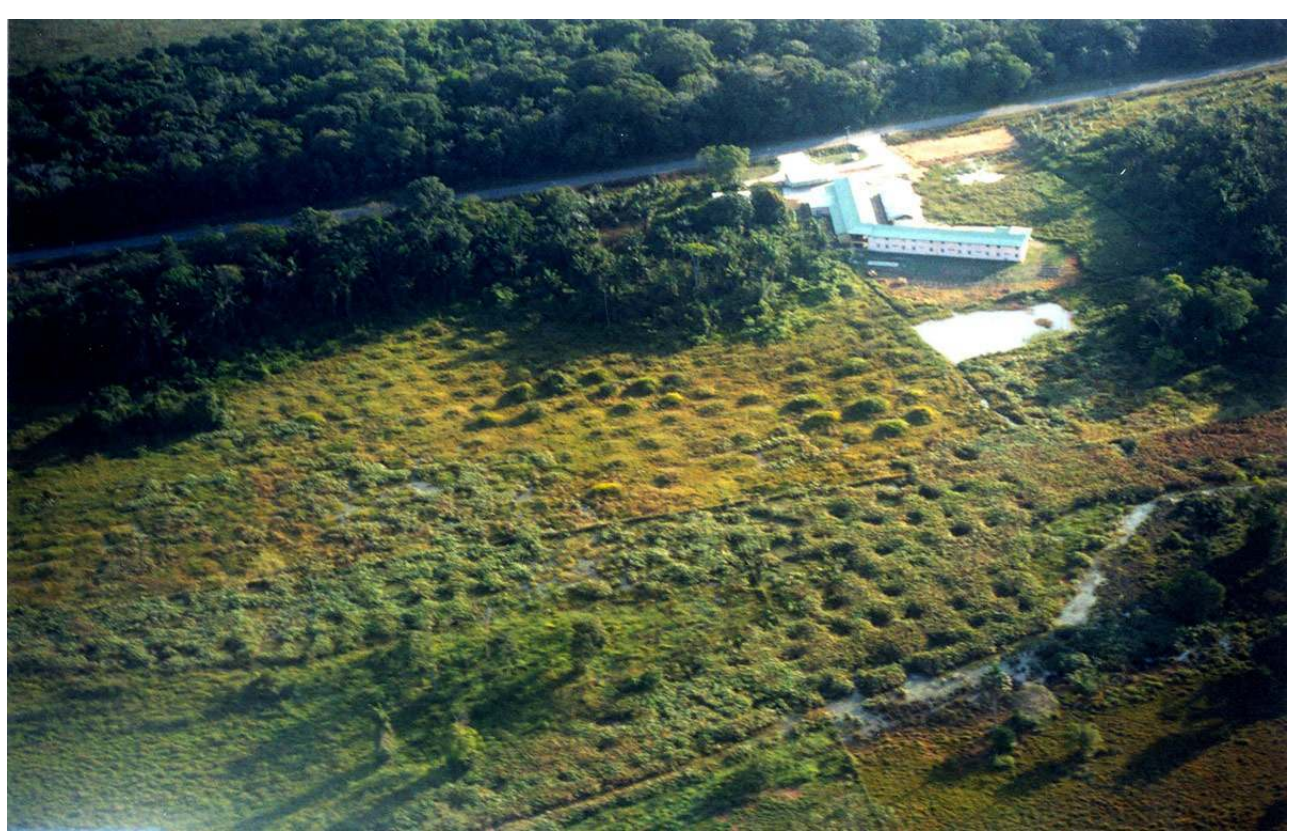

(c) S. Rostain

\section{Menaces sur les cheniers}

Les cordons sableux proches des champs surélevés ne sont pas exempts de menaces, et sont même plus en danger face à l'intervention humaine actuelle.

Le littoral des Guyanes fait partie de la vaste plaine sédimentaire Quaternaire qui s'étend sur plus de $1600 \mathrm{~km}$ entre l'embouchure de l'Amazone et le Delta de l'Orénoque. Elle mesure quelque $90 \mathrm{~km}$ de large dans le district de Nickerie au Suriname. En revanche, elle se rétrécit considérablement en Guyane française, n'atteignant que $5 \mathrm{~km}$ de largeur à l'ouest de l'île de Cayenne, et une moyenne de $16 \mathrm{~km}$ vers l'est. Cet environnement se caractérise par des savanes inondables et des marais, d'où émergent de-ci de-là quelques îlots forestiers et des cheniers (Figure 21). Ces derniers sont en réalité d'anciennes plages, constituées lors d'épisodes de recul du rivage et de dépôt de sable, essentiellement venu des fleuves locaux. Après, ces cordons se retrouvent enfermés dans la plaine littorale suite au dépôt de vases qui aboutit à la formation de nouveaux marais et, de manière plus générale, à l'accrétion progressive de la côte. Au bout du compte, plusieurs séries de cheniers sub-parallèles coupent les marécages côtiers, offrant d'étroits espaces exondés recouverts d'une dense forêt littorale.

Plusieurs sites archéologiques, souvent associés aux complexes de champs surélevés, ont été observés sur ces cheniers côtiers entre l'île de Cayenne et le Delta de l'Orénoque, territoire favori des terrassiers précolombiens (Figure 22). En Guyane française, face aux travaux modernes de plus en plus destructeurs, ces sites sont régulièrement fouillés, notamment grâce aux lois sur les interventions archéologiques préventives ou à des programmes de recherche comme celui mené par notre équipe (Figure 23). 
Figure 21 : Cheniers de la plaine côtière orientale du Suriname

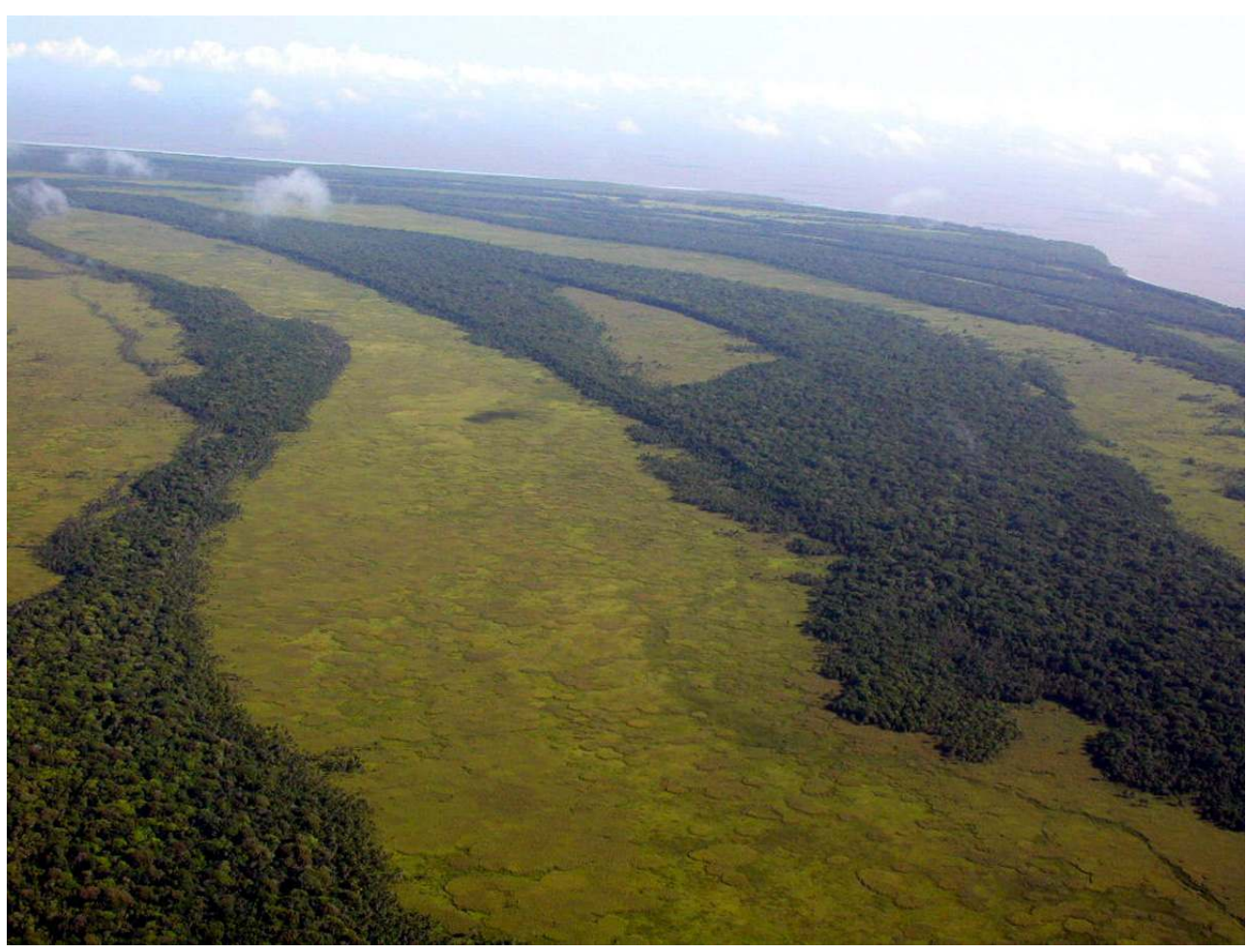

(C) S. Rostain

Figure 22 : Site archéologique de Bois Diable sur un chenier à la sortie de Kourou, Guyane française, associé à des champs surélevés précolombiens dans le marais adjacent et des canaux d'époque coloniale

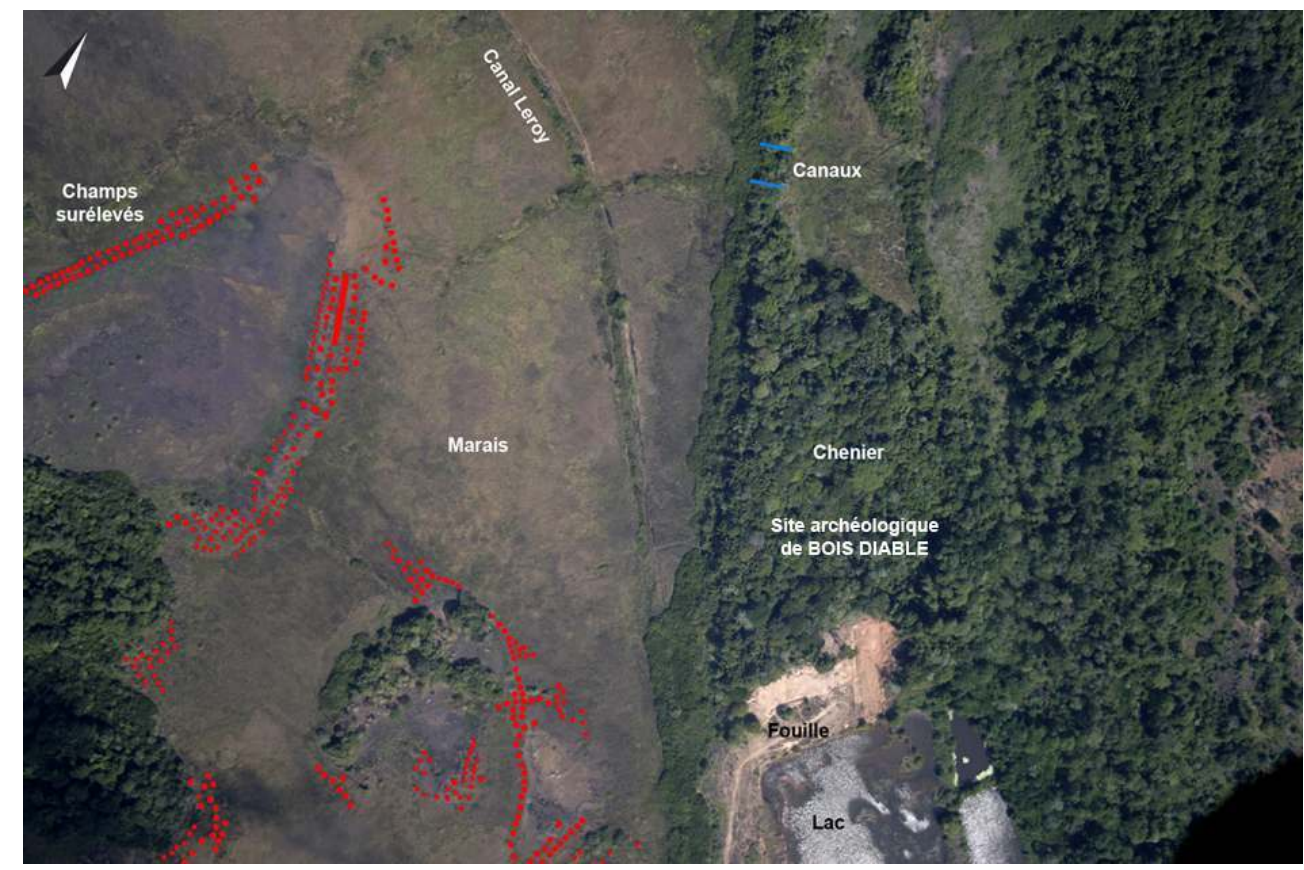

Photographie L'avion jaune et interprétation S. Rostain 
Figure 23 : Fouille du site archéologique de Sable Blanc en 2007, sur un chenier près d'Iracoubo, Guyane française

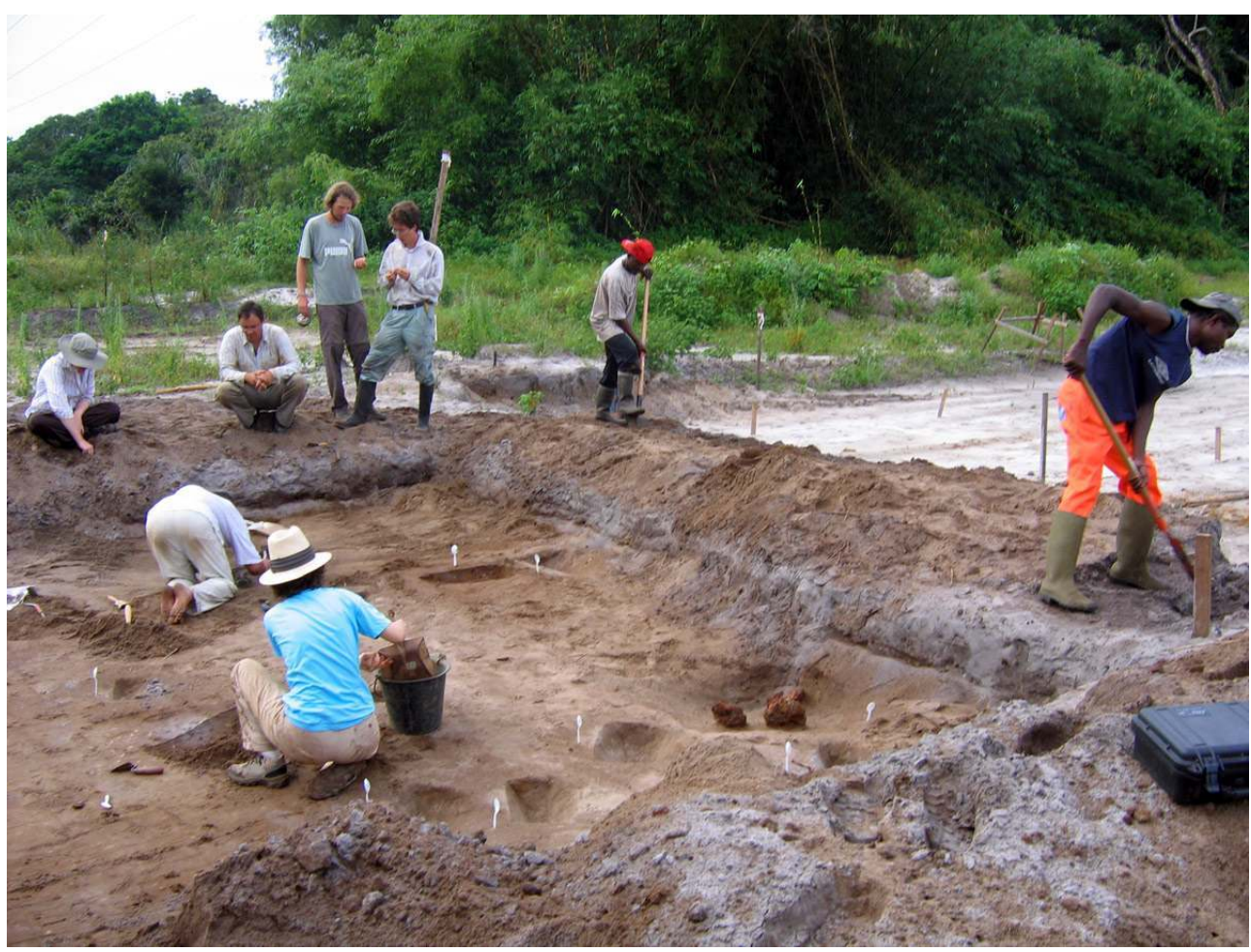

(c) S. Rostain

Logiquement, ces emplacements exondés et sains continuèrent à être privilégiés pour les établissements depuis la Conquête. L'accroissement récent de l'urbanisation et de la construction de routes sur le littoral des Guyanes, et plus particulièrement sur les cheniers, a modifié profondément le paysage originel. Si cela est évidemment très dommageable pour les sites archéologiques d'habitat qui "y» étaient implantés préférentiellement, cela affecte relativement peu les terrassements précolombiens, qui furent plutôt réalisés dans les aires inondables alentours.

Les marais ne sont pas pour autant moins préservés des menaces de destruction, car la récente accélération prodigieuse de constructions résidentielles en Guyane française envahit peu à peu les terrains marécageux. Pour se faire, les aires inondables sont progressivement remblayées avec du sable qui provient... des cheniers. En effet, depuis des années, de grandes sablières se chargent d'extraire du sable des cheniers pour gagner du terrain sur les marais afin d'édifier des routes ou des lotissements (Figure 24). La sablière de l'ouest de Kourou est probablement la plus représentative de ce type de dommage. Outre le fait qu'elle a annihilé le site archéologique de Bois Diable, attribué aux cultures Barbakoeba et Thémire (800-1600 apr. J.-C.), associé directement à des champs surélevés, le gisement fut exploité jusqu'aux années 1990 afin d'aménager les marécages au sud de la ville pour loger une population immigrante croissante, qui venait travailler pour le Centre Spatial Guyanais, dont l'activité s'amplifiait. Il en résulta une destruction importante du chenier dont les séquelles s'observent aujourd'hui sous la forme d'un lac artificiel (Figure 25). 
Figure 24 : Remblaiement de la savane inondable avec du sable provenant d'un chenier, près de Macouria, Guyane française

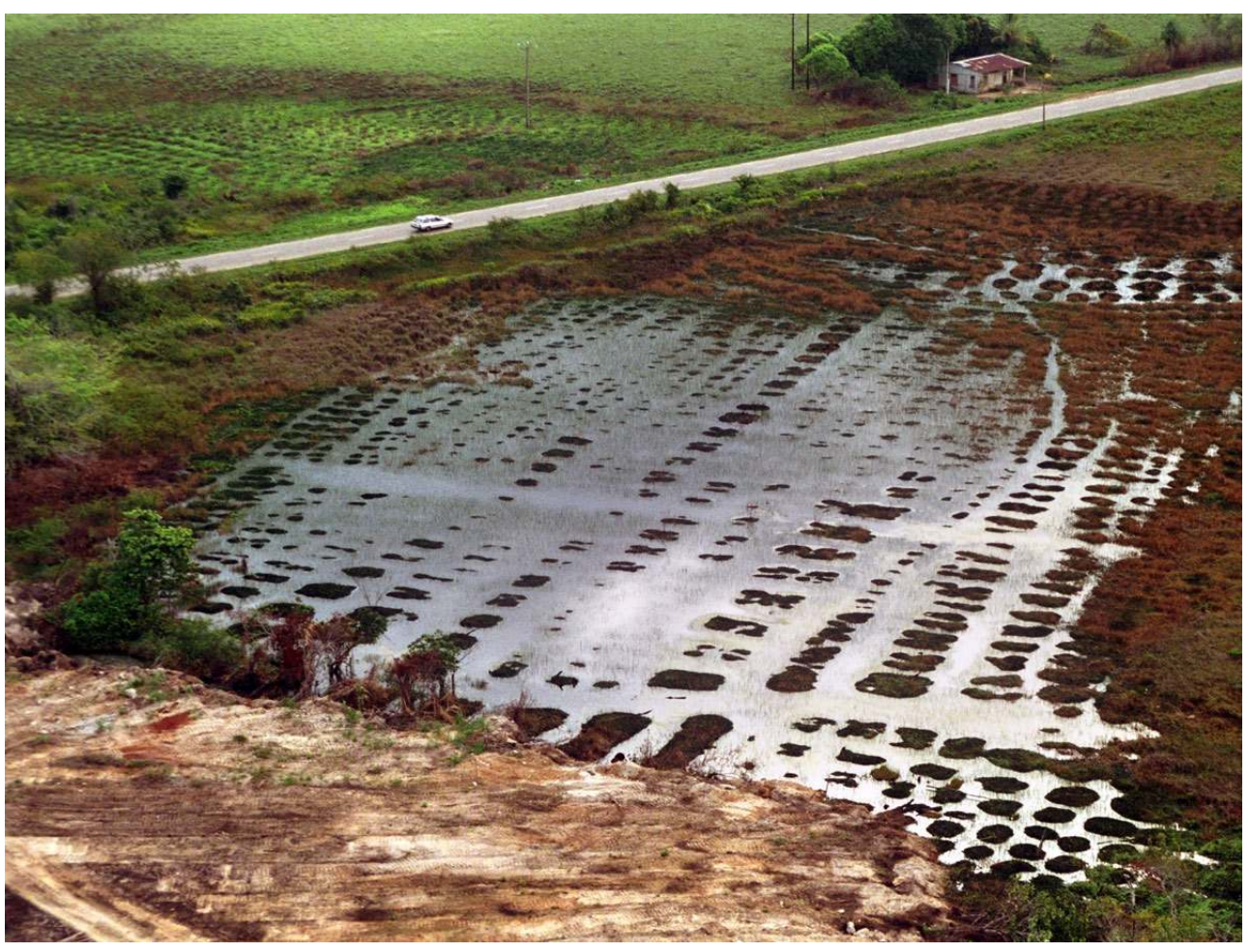

(c) S. Rostain

Figure 25 : Lac artificiel de Bois Diable, Guyane française, formé par l'extraction intensive de sable sur le chenier

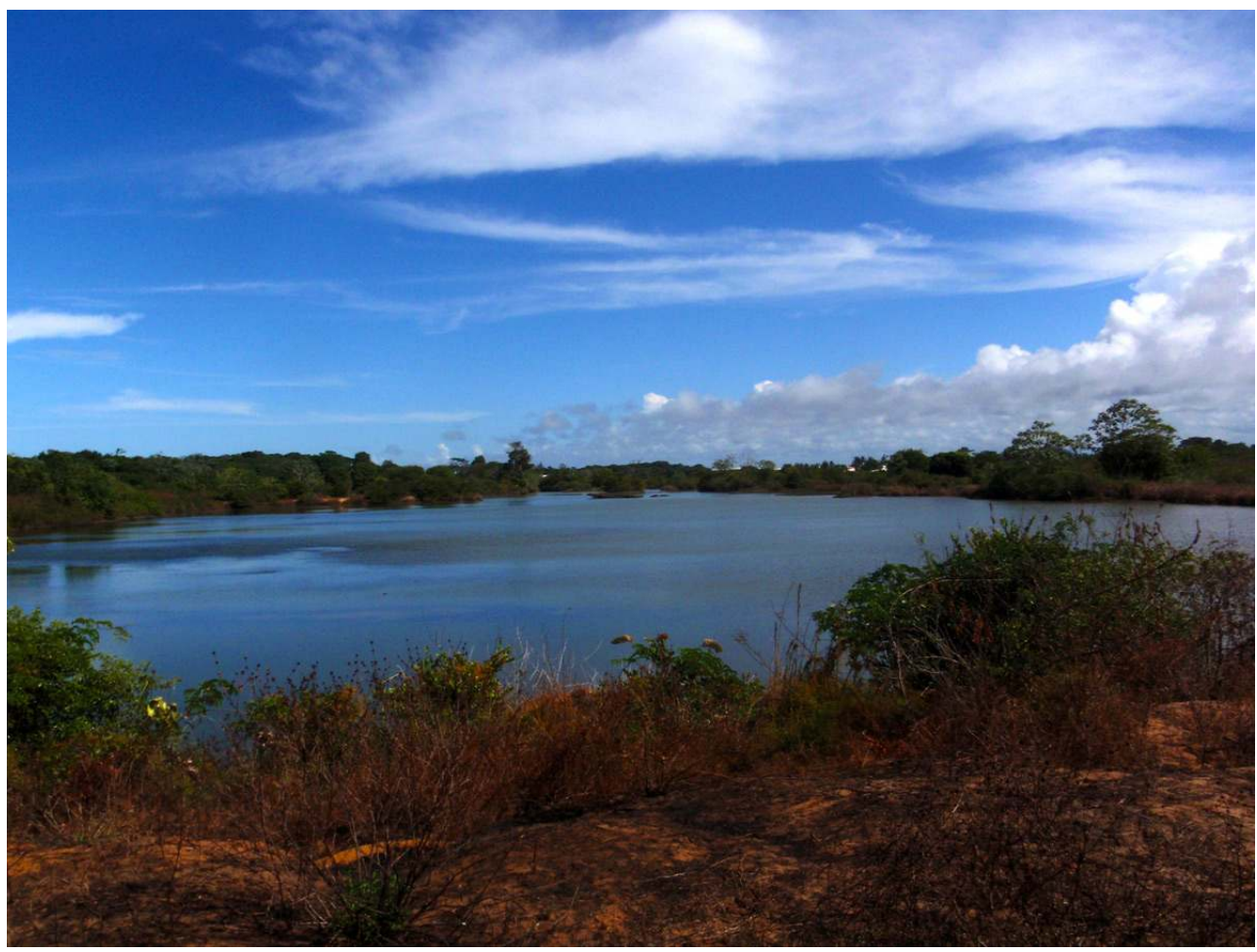

(c) S. Rostain 
et creusements provoquent également des modifications dans les drainages existant qui aboutissent à des colluvionnements nouveaux. On a ainsi cité auparavant le cas, à l'est de Kourou, d'un complexe de champs surélevés fermé par une digue artificielle édifiée par les agriculteurs précolombiens qui avait complètement disparu en quelques années.

\section{Protection des structures précolombiennes}

En général, les buttes de terre sont mieux préservées dans les aires forestières ou dans les milieux saisonnièrement inondés que dans les espaces ouverts et secs, comme le sommet herbeux des cordons sableux (Figure 26). À l'inverse, les structures creusées tendent à se conserver de meilleure manière dans des conditions opposées. Ainsi, les canaux s'effacent plus, sous l'apport de colluvionnements, dans les marais que sur les cheniers. Quoi qu'il en soit, on ne peut rester passif face aux agressions du monde moderne que subissent de plus en plus souvent ces vestiges.

Figure 26 : Complexe de champs surélevés apparu à la faveur d'un brûlis d'une aire de forêt qui l'avait jusqu'alors préservé, au nord de la Montagne des Pères, Guyane française

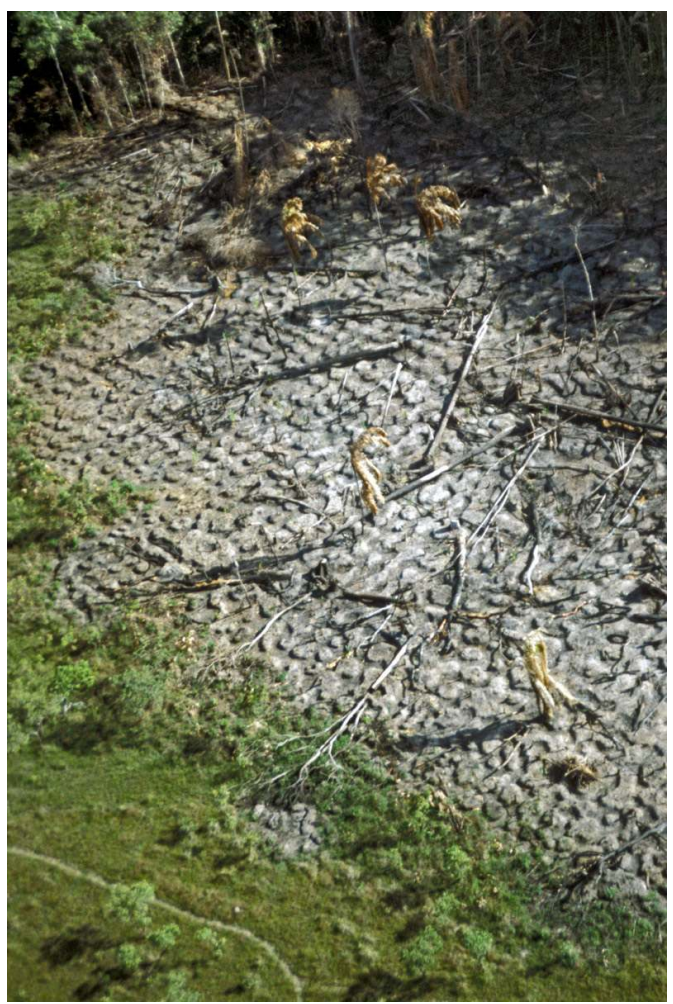

(c) S. Rostain

En tant qu'artefacts paysagers - on pourrait les appeler des «landfacts » -, les champs surélevés et les structures de terre associées devraient être protégés. Une telle mission incombe en général aux instances nationales du Patrimoine, tel le Service Régional d'Archéologie (SRA) en Guyane française. Malheureusement, aucune mesure de protection n'a été mise en place jusqu'à présent par ce service pour préserver ce patrimoine archéologique qui subit ainsi en toute impunité les attaques destructives des 
entrepreneurs et agriculteurs profitant de cette inaction. Le chercheur ne peut alors qu'assister, mousse et pampre, à l'anéantissement progressif de ce patrimoine millénaire.

Finalement, ce sont les réserves naturelles et les territoires contrôlés qui sont, pour l'instant, les plus efficaces garants de la préservation des structures précolombiennes. Si l'on pouvait craindre, il y a une trentaine d'années, que l'extension du Centre Spatial Guyanais (CSG), près de Kourou, puisse porter atteinte aux buttes, il n'en fut rien, bien au contraire. Grâce, d'une part, à une politique de limitation de grands travaux et de construction de bâtiments et, d'autre part, à l'interdiction au public de circuler dans les savanes sous l'emprise du CSG, les champs surélevés y ont été conservés bien mieux qu'ailleurs en Guyane française (Figure 27). La création récente du Parc Naturel régional de la Guyane est également bénéfique. Une grande portion du littoral occidental du pays est ainsi sous surveillance et, bien que les contrôles ne concernent pas les champs surélevés, ces derniers profitent de cette situation.

Figure 27 : Champs surélevés sur le territoire du Centre Spatial Guyanais, près de Kourou, Guyane française

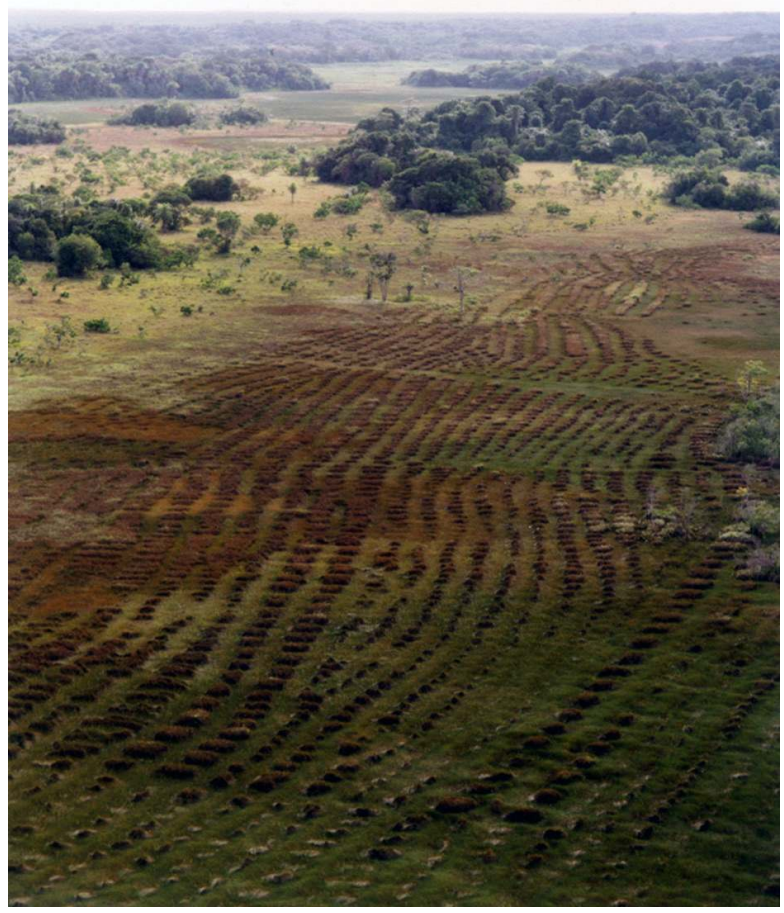

(c) S. Rostain

\section{Perspectives}

47 L'acceptation de l'origine et de la nature bioculturelles de paysages tels que les savanes côtières de Guyane est récente, car il faut rappeler la virulence de certains scientifiques il y a encore peu d'années qui ne voulaient pas admettre la moindre intervention humaine ancienne dans les environnements tropicaux. Ainsi, malgré l'accumulation de données de plus en plus nombreuses sur l'impact humain précolombien en Amazonie, la fameuse archéologue Betty Meggers nia toujours le poids de cette intervention, se cantonnant à un 
déterminisme écologique rigide. Elle republia ainsi au milieu des années 1990 son ouvrage contesté de 1971 (Meggers 1971) Amazonia: Man and culture in a counterfeit paradise (Meggers 1996) et y rajouta une postface polémique où elle tentait de démontrer l'impuissance de l'homme face à une nature contraire, l'expérience d'un géant industriel nord-américain ayant échoué dans une tentative d'exploiter extensivement des champs de caoutchouc dans le bas Amazone lui servant d'argument définitif. Plus près de nos considérations, la même auteure publia par la suite un article critique affirmant, entre autres, que les savanes à buttes sud-américaines pourraient être en réalité des formations totalement naturelles (Meggers 2003). Bien qu'il existe une diversité de telles savanes à buttes d'origine naturelle, on rencontre en même temps une diversité de vestiges de champs surélevés, et les organismes ingénieurs créateurs des buttes naturelles interagissent avec les actions des ingénieurs humains (McKey et al. 2014). Il est temps que cesse le dialogue de sourds entre archéologues et écologues au sujet de la nature biologique ou culturelle des paysages d'Amazonie, pour comprendre leur nature bioculturelle (Briggs et al. 2006 ; McKey et al. 2014).

Dans le même ordre d'idée, rares furent ceux qui acceptèrent d'emblée l'idée de constructions humaines lorsque les champs surélevés furent découverts en Guyane en 1989 (Rostain 1991). Les discussions furent âpres à l'époque, certains ne pouvant admettre l'idée d'une ingénierie précolombienne de haut niveau et ce n'est que face à l'accumulation de preuves inattaquables grâce au perfectionnement des techniques d'étude (modélisation spatiale, datations, restes fossiles des plantes cultivées, etc.) que les plus vindicatifs se résignèrent récemment.

49 En fait, un courant puissant naquit à la fin $d u x^{e}$ siècle pour rejeter toute forme d'ancienne influence humaine dans la constitution des paysages actuels. On se souviendra du livre "The Smithsonian Atlas of the Amazon» (Goulding et al. 2003) dans lequel les différents bassins constitutifs de l'Amazonie sont précisément décrits, mais où l'on ne trouve aucune mention des activités humaines actuelles et passées dans ce milieu tropical, ou même de l'homme tout court. Alors, qu'y a-t-il derrière cet acharnement virulent à nier l'action anthropique? Les raisons sont probablement multiples, et parfois inconscientes, mais inutiles de développer ici. Quoi qu'il en soit, il est aujourd'hui admis qu'il ne faut plus considérer l'Amazonie comme un simple gisement de ressources naturelles où l'on peut puiser impunément, les conséquences d'une telle exploitation pouvant être catastrophiques.

Par ailleurs, la valeur des sites archéologiques en termes de conservation n'est pas une question simple (Edson 2004; Karlström 2013). Selon Holtorf (2001), le passé est en quelque sorte une ressource renouvelable, car l'héritage est créé, détruit et recréé continuellement. Il remarque aussi que si les activités humaines modernes tels que la construction, le labourage, le feu, etc., menacent les sites (Figure 28), c'est souvent grâce à ces mêmes « menaces » que de nombreux sites archéologiques ont pu être découverts. Il argumente aussi qu'il est contreproductif d'essayer de protéger un nombre excessif de sites archéologiques. Pour l'Angleterre seule (et non pas l'ensemble de la GrandeBretagne), la gestion et la protection des 657000 sites archéologiques enregistrés (Holtorf 2001) dépasseraient de loin les ressources disponibles. Cependant, en ce qui concerne la conservation du patrimoine bioculturel du littoral guyanais, on est loin de cet état des lieux. Les vestiges des terrassements précolombiens risquent d'être effacés totalement si quelques sites ne bénéficient pas de protection urgente. 
Figure 28 : Cette photo aérienne d'un complexe de champs surélevés du centre de la Guyane française réunit les principaux dangers modernes les menaçant : les terrassements avec l'arasement de pelleteuse à gauche, l'élevage avec la barrière à bétail au milieu, l'incendie avec l'aire brûlée en bas, et les constructions avec la route et son fossé à droite

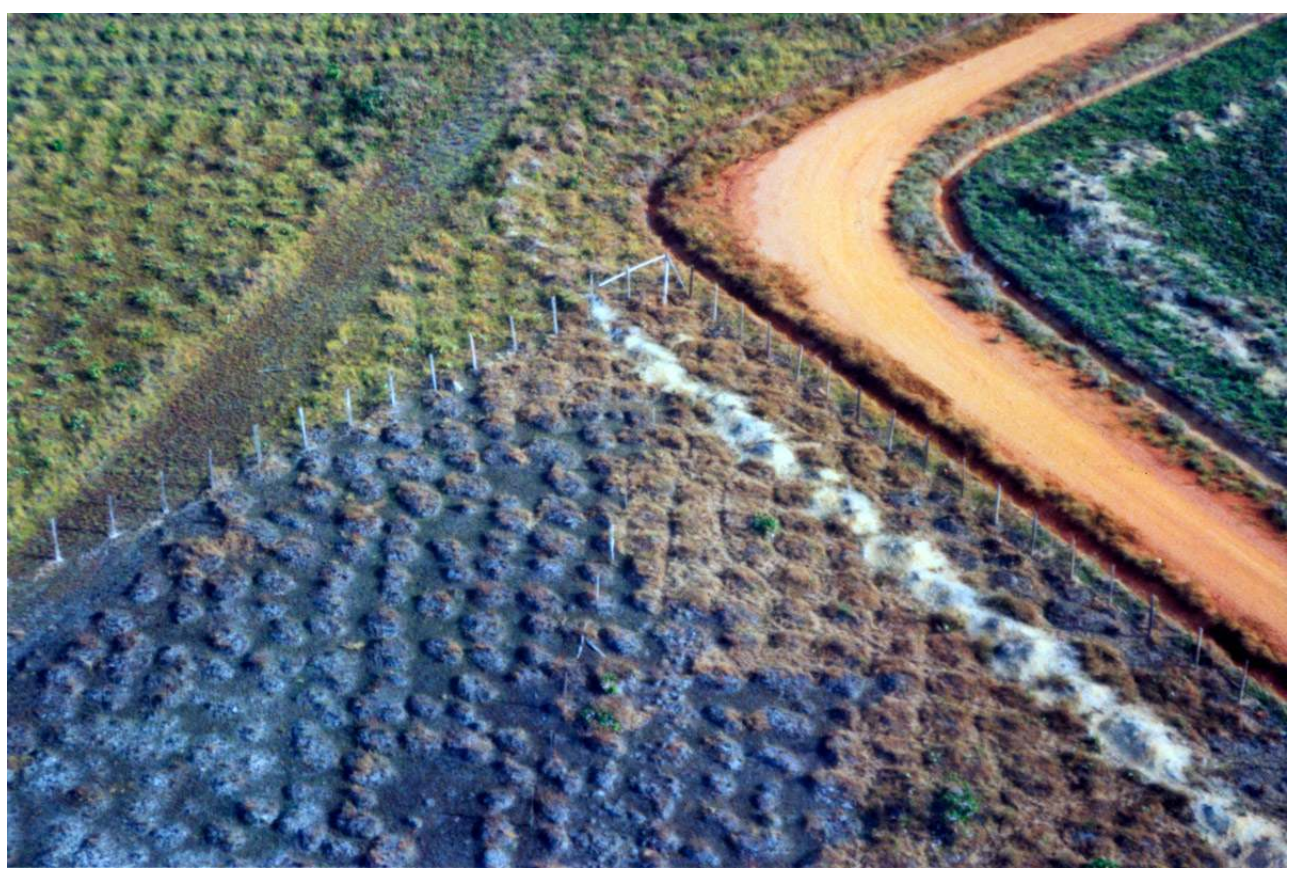

(c) S. Rostain

51 La reconnaissance du besoin de sauvegarder non seulement les bâtiments et sites historiques mais aussi les paysages culturels et bioculturels, est aussi une reconnaissance de l'inséparabilité de l'Homme et la nature. La préservation des paysages est ancrée dans la Convention Européenne du Paysage (Convention de Florence), dont la France est signataire (http://www.coe.int/t/dg4/cultureheritage/heritage/Landscape/ default_fr.asp). L'institution de cette convention a incité la préparation d'Atlas des Paysages pour différents départements, dont "L'Atlas des Paysages de Guyane », qui reconnaît les paysages marqués par les vestiges de champs surélevés précolombiens comme partie intégrante de l'empreinte humaine en Guyane (DEAL Guyane 2013 : 25). Malgré cette reconnaissance officielle, et en dépit du fait que les anciens champs surélevés et autres «bas-fonds à buttes " sur le littoral guyanais hébergent une grande biodiversité végétale (Léotard 2012), ces paysages sont largement « sous le radar » des conservationnistes, des gestionnaires des aires protégées, et des citoyens. Nous ne connaissons aucun sentier interprétatif, ni jardin de démonstration, qui pourrait faire vivre ces paysages dans le conscient collectif d'aujourd'hui. Malgré quelques timides tentatives à Sinnamary et Kourou, les débouchés touristiques semblent encore bien utopiques, mais sont-ils réellement souhaitables (voir Jansen-Verbeke \& McKercher [2013]) ? En insistant sur leur intérêt - comme témoins de l'histoire humaine de la Guyane, comme exemples d'un type d'agriculture qui travaille avec les zones humides et non pas contre elles - et en documentant les activités humaines qui risquent d'effacer leurs traces, nous espérons inciter des efforts institutionnels pour la conservation de ce patrimoine bioculturel.

52 Les savanes littorales guyanaises sont un héritage bioculturel complexe issu d'actions séculaires variées qu'il est indécent d'ignorer. Les pays du Sud ont déjà commencé à 
prendre conscience du besoin de changer notre regard et notre attitude sur les paysages qui nous entourent. L'Équateur fut ainsi la première contrée à voter, en 2008, une constitution, notamment sous la pression d'une menace alors croissante d'exploitation pétrolière anarchique, pour attribuer à la forêt tropicale, aux îles, aux rivières et même à l'air, les mêmes droits qu'aux hommes. En Nouvelle-Zélande, la rivière Whanganui a obtenu en 2012 une identité légale, qui lui octroie les mêmes droits qu'une personne devant la justice. Évidemment, on peut disserter longuement sur la validité de reconnaître ou non que les paysages aient des droits, il n'en demeure pas moins qu'il faut désormais admettre que, vis-à-vis de cette nature, c'est l'homme qui a des devoirs.

\section{BIBLIOGRAPHIE}

Baer A., Ladefoged T.N., Stevenson C.M. \& Haoa S. 2008 - The surface rock gardens of prehistoric Rapa Nui. Rapa Nui Journal $22: 102-109$.

Balée W.L. \& Erickson C.L. (Ed.) 2006 - Time and complexity in historical ecology: studies in the Neotropical lowlands. New York, Columbia University Press, 17 p.

Barrutia O., Artetxe U., Hernández A., Olano J.M., García-Plazaola J.I., Garbisu C. \& Becerril J.M. 2011 - Native plant communities in an abandoned Pb-Zn mining area of northern Spain: Implications for phytoremediation and germplasm preservation. International Journal of Phytoremediation $13: 256-270$.

Baveye P.C. 2013 - Comment on "Ecological engineers ahead of their time: The functioning of pre-Columbian raised-field agriculture and its potential contributions to sustainability today" by Dephine Renard et al. Ecological Engineering 52 : 224-227.

Briggs J.M., Spielmann K.A., Schaafsma H., Kintigh K.W., Kruse M., Morehouse K. \& Schollmeyer K. 2006 - Why ecology needs archaeologists and archaeology needs ecologists. Frontiers in Ecology and the Environment $4: 180-188$.

DEAL (Direction de l'Environnement, de l'Aménagement et du Logement) Guyane 2013 - L'Atlas des Paysages de Guyane. http://www.paysagesdeguyane.fr/

Denevan W.M. 2001 - Cultivated landscapes of native Amazonia and the Andes. New York, Oxford University Press, 396 p.

Denevan W.M. \& Turner B.L. 1974 - Forms, functions and associations of raised fields in old world tropics. Journal of Tropical Geography 39 : 24-33.

Diamond J. 2005 - Collapse: How societies choose to fail or succeed. New York, Penguin Books, 592 p.

Edson G. 2004 - Heritage: Pride or passion, product or service? International Journal of Heritage Studies 10 : 333-348.

Erickson C.L. 2003 - Agricultural landscapes as world heritage: raised field agriculture in Bolivia and Peru. In Teutonico J.M. \& Matero F. (Ed.) Managing change: Sustainable approaches to the conservation of the built environment. Los Angeles, Getty Conservation Institute : 181-204. 
Erickson C. 2006 - El valor actual de los camellones de cultivo precolombinos: Experiencias del Perú y Bolivia. In Valdez F. (Ed.) Agricultura ancestral. Camellones y albarradas: Contexto social, usos y retos del pasado y del presente. Quito, Ediciones Abya-Yala : 315-339.

Fischenich J.C. 2003 - Technical considerations for evaluating riverine/riparian restoration projects. ERDC/EL TR-WRAP-03-XX, April 2003, U.S. Army Engineers, Research and Development Center Environmental Laboratory, Vicksburg, MS, USA.

Fisher C.T. \& Feinman G.M. 2005 - Introduction to « landscapes over time ». American Anthropologist $107: 62-69$.

Fuller T. \& Qingwen M. 2013 - Understanding Agricultural Heritage Sites as complex adaptive systems: the challenge of complexity. Journal of Resources and Ecology 4 : 195-201.

Glaser B. \& Birk J.J. 2012 - State of the scientific knowledge on properties and genesis of Anthropogenic Dark Earths in Central Amazonia (terra preta de Índio). Geochimica et Cosmochimica Acta $82: 39-51$.

Goulding M., Barthem R. \& Ferreira E. 2003 - The Smithsonian Atlas of the Amazon. Washington, D.C. \& London, Smithsonian Books, 256 p.

Hayashida F.M. 2005 - Archaeology, ecological history, and conservation. Annual Review of Anthropology 34 : 43-65.

Holtorf C.J. 2001 - Is the past a non-renewable resource? In Layton R., Stone P.G. \& Thomas J. (Ed.) Destruction and conservation of cultural property. London \& New York, Routledge : 286-297.

Hunt T.L. 2006 - Rethinking the fall of Easter Island. American Scientist 94 : 412-419.

Hunt T. \& Lipo C. 2011 - The statues that walked: unraveling the mystery of Easter Island. New York, Simon and Schuster, $256 \mathrm{p}$.

Iriarte J., Glaser B., Watling J., Wainwright A., Birk J.J., Renard D., Rostain S. \& McKey D. 2010 - Late Holocene Neotropical agricultural landscapes: phytolith and stable carbon isotope analysis of raised fields from French Guianan coastal savannahs. Journal of Archaeological Science 37 : 2984-2994.

Iriarte J., Power M.J., Rostain S., Mayle F.E., Jones H., Watling J., Whitney B.S. \& McKey D.B. 2012 - Fire-free land use in pre-1492 Amazonian savannas. Proceedings of the National Academy of Sciences, USA 109 : 6473-6478.

Jansen-Verbeke M. \& McKercher B. 2013 - Reflections on the myth of tourism preserving "traditional" agricultural landscapes. Journal of Resources and Ecology 4 : 242-249.

Jones C.G., Lawton J.H. \& Shachak M. 1994 - Organisms as ecosystem engineers. Oikos 69 : 373-386. Karlström A. 2013 - Local heritage and the problem with conservation. In Brockwell S., O'Connor S. \& Byrne D. (Ed.) Transcending the culture: nature divide in cultural heritage. Views from the AsiaPacific region. Canberra, ANU E Press : 141-155.

Koohafkan P., \& Altieri M.A. 2011 - Globally Important Agricultural Heritage Systems (GIAHS). A legacy for the future. Rome, FAO, $41 \mathrm{p}$.

Léotard G. 2012 - Projet LIFE+Cap DOM : Étude botanique des savanes de Guyane - Rapport final. GEPOG, $125 \mathrm{p}$.

Lipe W.D. 2002 - Public benefits of archaeological research. In Little B. (Ed.) Public Benefits of Archaeology. Gainesville, University Press of Florida : 20-28. 
Lombardo U., Canal-Beeby E., Fehr S. \& Veit H. 2011 - Raised fields in the Bolivian Amazonia: a prehistoric green revolution or a flood risk mitigation strategy? Journal of Archaeological Science $38: 502-512$.

Losfeld G., Mathieu R., L'Huillier L., Fogliani B., Jaffré T. \& Grison C. 2015 - Phytoextraction from mine spoils: insights from New Caledonia. Environmental Science and Pollution Research 22 (8) : 5608-5619.

Mann C.C. 2005 - 1491: New revelations of the Americas before Columbus. New York, Knopf, 541 p.

Martin J.F., Roy E.D., Diemont S.A. \& Ferguson B.G. 2010 - Traditional ecological knowledge (TEK): ideas, inspiration, and designs for ecological engineering. Ecological Engineering $36: 839-849$.

McKey D., Durécu M., Solibiéda A., Raimond A., Adame Montoya K.L., Iriarte J., Renard D., Suarez Jimenez L.E., Rostain S. \& Zangerlé A. 2014 - New approaches to pre-Columbian raised-field agriculture: ecology of seasonally flooded savannas, and living raised fields in Africa, as windows on the past and the future. In Rostain S. (Ed.) Amazonía. Memorias de las conferencias magistrales del 3er Encuentro Internacional de Arqueología Amazónica, MCCTH/SENESCYT/3EIAA, Quito : 91-136.

McKey D., Rostain S., Iriarte J., Glaser B., Birk J., Holst I. \& Renard D. 2010 - Pre-Columbian agricultural landscapes, ecosystem engineers and self-organized patchiness in Amazonia. Proceedings of the National Academy of Sciences, USA 107 : 7823-7828.

Meggers B.J. 1971 - Amazonia: Man and culture in a counterfeit paradise. Chicago, Aldine, vii-182 p.

Meggers B.J. 1996 - Amazonia: Man and culture in a counterfeit paradise (revised edition), Washington, London, Smithsonian Institution Press, 214 p.

Meggers B.J. 2003 - Natural versus anthropogenic sources of Amazonian biodiversity: the continuous quest for El Dorado. In Bradshaw G.A. \& Marquet P.A. (Ed.) How Landscapes Change. Berlin, Springer-Verlag : 89-107.

Merlín-Uribe Y., González-Esquivel C.E., Contreras-Hernández A., Zambrano L., Moreno-Casasola P. \& Astier M. 2013 - Environmental and socio-economic sustainability of chinampas (raised beds) in Xochimilco, Mexico City. International Journal of Agricultural Sustainability $11: 216-233$.

Mulrooney M.A. 2013 - An island-wide assessment of the chronology of settlement and land use on Rapa Nui (Easter Island) based on radiocarbon data. Journal of Archaeological Science 40 : 4377-4399.

Niederberger Betton C. 1987 - Paléopaysages et archéologie pré-urbaine du bassin du Mexique. Mexico, CEMCA, $357 \mathrm{p}$.

Pélissier P. 1966 - Les paysans du Sénégal. Les civilisations agraires du Cayor à la Casamance. SaintYrieix, France, Imprimerie Fabrègue, 941 p.

Pfahler V., Glaser B., McKey D. \& Klemt E. 2015 - Soil redistribution in abandoned raised fields in French Guiana assessed by radionuclides. Journal of Plant Nutrition and Soil Science. Online early. DOI: $10.1002 /$ jpln.201400279

Rascio N. \& Navari-Izzo F. 2011 - Heavy metal hyperaccumulating plants: how and why do they do it? And what makes them so interesting? Plant Science $180: 169-181$.

Renard D., Birk J.J., Zangerlé A., Lavelle P., Glaser B., Blatrix R. \& McKey D. 2013 - Ancient human agricultural practices can promote activities of contemporary non-human soil ecosystem engineers: A case study in coastal savannas of French Guiana. Soil Biology and Biochemistry 62 : 46-56. 
Renard D., Iriarte J., Birk J.J., Rostain S., Glaser B. \& McKey D. 2012 - Ecological engineers ahead of their time: The functioning of pre-Columbian raised-field agriculture and its potential contributions to sustainability today. Ecological Engineering 45 : 30-44.

Rick T.C. \& Lockwood R. 2013 - Integrating paleobiology, archeology, and history to inform biological conservation. Conservation Biology 27 : 45-54.

Rostain S. 1991 - Les champs surélevés amérindiens de la Guyane. Cayenne, Centre ORSTOM de Cayenne ; Institut Géographique National. (La Nature et l'Homme), 28 p.

Rostain S. 2008 - Agricultural earthworks on the French Guiana coast. In Silverman H. \& Isbell W. (Ed.) Handbook of South American archaeology. New York Springer ; Kluwer ; Plenum : 217-234.

Rostain S. 2010 - Pre-Columbian earthworks in coastal Amazonia. Diversity 2 : 353-369.

Rostain S. 2012a - Islands in the rainforest. Landscape management in pre-Columbian Amazonia. Series “New Frontiers in Historical Ecology”. Walnut Creek, CA, USA, Left Coast Press, 277 p.

Rostain S. 2012b - Between Sierra and Selva: pre-Columbian landscapes in the upper Ecuadorian Amazonia. Quaternary International 249 : 31-42.

Rostain S. 2014 - The Guianas, pre-Columbian heritage. In Smith C., Silverman H. \& Kynourgiopoulou V. (Ed.) Encyclopedia of global archaeology, section “World Heritage”. New York, Springer : 7293-7302.

Rull V., Cañellas-Boltà N., Sáez A., Margalef O., Bao R., Pla-Rabes S., Valero-Garcés B. \& Giralt S. 2013 - Challenging Easter Island's collapse: the need for interdisciplinary synergies. Frontiers in Ecology and Evolution 1. doi: 10.3389/fevo.2013.00003.

Ryan M.M. 2009 - The house that Smokey built: The Forest Service management of historic structures in wilderness. Doctoral dissertation, Missoula, MT, USA, University of Montana, 104 p. http:// etd.lib.umt.edu/theses/available/etd-07162009-095329/

Sandor J.A. \& Furbee L. 1996 - Indigenous knowledge and classification of soils in the Andes of Southern Peru. Soil Science Society of America Journal 60 : 1502-1512.

Scharf E.A. 2014 - Deep time: the emerging role of archaeology in landscape ecology. Landscape Ecology 29 : 563-569.

Schmidt M. 2013 - Amazonian Dark Earths: pathways to sustainable development in tropical rainforests? Boletim do Museu Paraense Emílio Goeldi. Ciências Humanas 8 : 11-38.

Spencer C.S., Redmond E.M. \& Rinaldi M. 1994 - Drained fields at La Tigra, Venezuelan Llanos: a regional perspective. Latin American Antiquity 5 : 95-110.

Stump D. 2013 - On applied archaeology, indigenous knowledge, and the usable past. Current Anthropology 54 : 268-298.

Teixeira W.G., Kern D.C., Madari B.E., Lima H.N. \& Woods W. (Ed.) [2010] - As terras pretas de Indio da Amazônia: sua caracterização e uso deste conhecimento na criação de novas areas. Manaus, Brésil, Editora da Universidade Federal do Amazonas/Embrapa Amazônia Ocidental, 419 p.

The National Planning Office of Suriname \& Regional Development and Physical Planning Department 1988 - Suriname Planatlas. Washington D.C, Organization of American States, Department of Regional Planning.

Transler A.-L., Saffache P. \& Moullet D. 2006 - Impacts des activités agricoles sur le littoral guyanais. La Géographie, spécial « Antilles-Guyane », hors-série 1522. Paris, Société de Géographie : 70-77. 
Vamerali T., Bandiera M. \& Mosca G. 2010 - Field crops for phytoremediation of metalcontaminated land. A review. Environmental Chemistry Letters $8: 1-17$.

Villars R. 1914 - Les colons de l'île verte. Paris, Société française d'imprimerie et de librairie, 320 p.

Walker J.H. 2004 - Agricultural change in the Bolivian Amazon. Pittsburgh/Trinidad, Fundación Kenneth Lee. (University of Pittsburgh Memoirs in Latin American Archaeology ; 13).

Xie J., Hu L., Tang J., Wu X., Li N., Yuan Y., Yanga H., Zhangb J., Luo S. \& Chen X. 2011 - Ecological mechanisms underlying the sustainability of the agricultural heritage rice-fish coculture system. Proceedings of the National Academy of Sciences, USA 108 : E1381-E1387.

\section{NOTES}

1. Par "savane ", on entend ici les savanes sèches, souvent inondables, ainsi que les marais qui les bordent. En Guyane française, ils représentent quelque 445000 hectares.

\section{RÉSUMÉS}

Les savanes côtières des Guyanes abritent des aires étendues couvertes de vestiges de l'agriculture précolombienne sur champs surélevés. Des études récentes ont démontré leur valeur pour la documentation de l'histoire de l'occupation humaine et des environnements de cette région. Dans le cas de la Guyane française, peu d'habitants actuels sont informés de l'existence de ces vestiges, et ceux qui le sont possèdent une compréhension limitée de leur signification. De fait, les divers dangers auxquels ce patrimoine bioculturel doit faire face ont largement été ignorés et non discutés. Peu de vestiges de champs surélevés ont été inclus dans les aires protégées. L'objectif de cet article est de documenter la richesse de ces paysages, d'illustrer leur valeur, et d'attirer l'attention sur les dangers qu'ils affrontent. Nous affirmons qu'ils méritent une meilleure protection.

The coastal savannas of the Guianas harbor extensive areas covered with vestiges of preColumbian raised-field agriculture. Recent studies have shown their value in documenting the history of human occupation and environments in the region. In the case of French Guiana, few of the present-day inhabitants are aware of the existence of these vestiges, and those who are have little appreciation of their significance. Thus the manifold dangers this biocultural heritage faces have largely gone unnoticed and unchallenged, and few raised-field vestiges are included in protected areas. The objective of this paper is to document the richness of these landscapes, illustrate their value, and draw attention to the threats facing them. We argue that they merit greater protection. 
INDEX

Index géographique : Guyane française

Keywords : agricultural heritage, archaeology, biocultural heritage, French Guiana, historical ecology, landscape ecology

Mots-clés : archéologie, écologie du paysage, écologie historique, patrimoine agricole, patrimoine bioculturel

\section{AUTEURS}

\section{STÉPHEN ROSTAIN}

ArchAm UMR 8096, CNRS, Maison René Ginouvès, 21 allée de l'université, 92323 Nanterre cedex

\section{DOYLE MCKEY}

CEFE UMR 5175, CNRS - Université de Montpellier - Université Paul-Valéry - EPHE - 1919 route de Mende, 34293 Montpellier cedex 5

Institut Universitaire de France 